\title{
LOCAL AND NONLOCAL CONTINUUM LIMITS OF ISING-TYPE ENERGIES FOR SPIN SYSTEMS*
}

\author{
ROBERTO ALICANDRO ${ }^{\dagger}$ AND MARIA STELLA GELLI ${ }^{\ddagger}$
}

\begin{abstract}
We study, through a $\Gamma$-convergence procedure, the discrete to continuum limit of Ising-type energies of the form $F_{\varepsilon}(u)=-\sum_{i, j} c_{i, j}^{\varepsilon} u_{i} u_{j}$, where $u$ is a spin variable defined on a portion of a cubic lattice $\varepsilon \mathbb{Z}^{d} \cap \Omega, \Omega$ being a regular bounded open set, and valued in $\{-1,1\}$. If the constants $c_{i, j}^{\varepsilon}$ are nonnegative and satisfy suitable coercivity and decay assumptions, we show that all possible $\Gamma$-limits of surface scalings of the functionals $F_{\varepsilon}$ are finite on $B V(\Omega ;\{ \pm 1\})$ and of the form $\int_{S_{u}} \varphi\left(x, \nu_{u}\right) d \mathcal{H}^{d-1}$. If such decay assumptions are violated, we show that we may approximate nonlocal functionals of the form $\int_{S_{u}} \varphi\left(\nu_{u}\right) d \mathcal{H}^{d-1}+\int_{\Omega} \int_{\Omega} K(x, y) g(u(x), u(y)) d x d y$. We focus on the approximation of two relevant examples: fractional perimeters and Ohta-Kawasaki-type energies. Eventually, we provide a general criterion for a ferromagnetic behavior of the energies $F_{\varepsilon}$ even when the constants $c_{i, j}^{\varepsilon}$ change sign. If such a criterion is satisfied, the ground states of $F_{\varepsilon}$ are still the uniform states 1 and -1 and the continuum limit of the scaled energies is an integral surface energy of the form above.
\end{abstract}

Key words. atomistic-to-continuum limit, $\Gamma$-convergence, spin systems, surface energies

AMS subject classification. 49J45

DOI. $10.1137 / 140997373$

1. Introduction. The description and prediction of mesoscopic pattern formation have been and continue to be an object of extensive research both in the physical and in the mathematical literature. A possible approach in pursuing this task, usually adopted in statistical mechanics, is the analysis of discrete models, which aims at predicting such patterns starting from discrete systems of particles in interaction. A different but somehow complementary approach is the analysis of continuum models, which very often correspond to a coarse-graining description of the previous ones, which averages or smooths away fine details but is capable of capturing some of the main features of the original problems.

Among the discrete models an efficient and celebrated example is given by the Ising model, introduced originally to model ferromagnetism in statistical mechanics but then applied successfully in many other contexts. In its original form it amounts to considering a Hamiltonian of the form (where n.n. stands for nearest neighbor)

$$
F(u)=-\sum_{n . n .} u_{i} u_{j}
$$

which is the energy of a system of interacting spins through their nearest-neighbor bonds. Here the spin variable $u$ is defined on a portion $\mathcal{L}$ of a periodic lattice, say $\mathbb{Z}^{d}$, and takes values in $\{-1,1\}$. By scaling $\mathbb{Z}^{d}$ by a small parameter $\varepsilon>0$ and identifying $\mathcal{L}$ with $\varepsilon \mathbb{Z}^{d} \cap \Omega$, where $\Omega$ is an open bounded open set of $\mathbb{R}^{d}$, it can be proved that

* Received by the editors November 25, 2014; accepted for publication (in revised form) December 30, 2015; published electronically March 10, 2016. The research of the authors have been supported by the Gruppo Nazionale per l'Analisi Matematica, la Probabilità e le loro Applicazioni (GNAMPA) of the Istituto Nazionale di Alta Matematica (INdAM).

http://www.siam.org/journals/sima/48-2/99737.html

†DIEI, Università di Cassino e del Lazio Meridionale, 03043 Cassino (FR), Italy (alicandr@ unicas.it).

${ }^{\ddagger}$ Dipartimento di Matematica, Università di Pisa, 56127 Pisa, Italy (gelli@dm.unipi.it). 
the discrete-to-continuum limit of suitable power scaling of $E$, as $\varepsilon \rightarrow 0$, resembles that of a Cahn-Hilliard-type functional. Namely, it leads in the limit as $\varepsilon \rightarrow 0$ to the surface energy $\int_{S_{u}}\left|\nu_{u}\right|_{1} d \mathcal{H}^{d-1}$ for $u \in B V(\Omega ;\{ \pm 1\})$, where $S_{u}$ is the jump set of $u$, that is the interface between the $\{u=1\}$ phase and the $\{u=-1\}$ phase, $\nu_{u}$ is the unit normal to $S_{u}$, and $|\cdot|_{1}$ is the $\ell^{1}$-norm in $\mathbb{R}^{d}$.

Ising-type energies are more generally written in the form

$$
F(u)=-\sum_{i \neq j \in \mathcal{L}} c_{i, j} u_{i} u_{j}
$$

Here, according to the sign of the constants $c_{i, j}$, we speak of ferromagnetic interactions, corresponding to $c_{i, j}>0$ and favoring uniform pairs $u_{i}=u_{j}$, or of antiferromagnetic interactions, corresponding to $c_{i, j}<0$ and preferring instead alternating pairs $u_{i}=-u_{j}$. Many studies have shown that models involving energies of the form (1.2), for a suitable choice of the range of interactions and of the sign of the constants $c_{i, j}$, can be approximated in the continuum by models involving surface energies of the form above, where the limiting interfaces may represent phase or antiphase boundaries (see [3] for some relevant examples in a variational framework).

A general question is, then, whether an approximation by surface energies can be used. The purpose of this paper is to answer this question and, more precisely, to determine a large class of energies of the form (1.2) whose discrete-to-continuum limits can be described by surface functionals.

We start our analysis by considering a bulk scaling of the functionals in (1.2) and letting the constants $c_{i, j}$ depend also on the small parameter $\varepsilon$. Therefore we consider energies of the form

$$
F_{\varepsilon}(u)=-\sum_{i \neq j \in \Omega_{\varepsilon}} \varepsilon^{d} c_{i, j}^{\varepsilon} u_{i} u_{j}
$$

defined for $u: \varepsilon \mathbb{Z}^{d} \cap \Omega \rightarrow\{-1,1\}$, where we have used the notation $\Omega_{\varepsilon}:=\mathbb{Z}^{d} \cap\left(\varepsilon^{-1} \Omega\right)$, $u_{i}:=u(\varepsilon i)$. The asymptotic behavior as $\varepsilon \rightarrow 0$ of energies of the form (1.3) has been studied in [5] and leads in the continuum limit to a class of integral functionals of the type

$$
\int_{\Omega} f(x, u(x)) d x
$$

where the limit variable $u$ represents the magnetization of the spin variable $u_{\varepsilon}$, that is, loosely speaking, $u(x)$ is the average value of $u_{\varepsilon}$ around $x$. We underline that, with this scaling, the asymptotic analysis gives some insight into the structure of the ground states only when the constants $c_{i, j}^{\varepsilon}$ change sign, leading to a competition between ferromagnetic and antiferromagnetic interactions.

Let us focus instead on the purely ferromagnetic case, i.e., assume $c_{i, j}^{\varepsilon} \geq 0$ for all $i, j \in \Omega_{\varepsilon}$. Note that with this assumption the ground states of $F_{\varepsilon}$ are trivially the constant functions $u \equiv 1$ and $u \equiv-1$. Nevertheless, if boundary conditions or additional constraints are added, minimizers are not trivial and it is interesting to describe their behavior as $\varepsilon \rightarrow 0$, i.e., when the number of indices diverge. To this end, a higher order description of $F_{\varepsilon}$ is needed.

Let $m_{\varepsilon}:=\min F_{\varepsilon}=F_{\varepsilon}( \pm 1)$ and consider a surface scaling of $F_{\varepsilon}$ given by

$$
E_{\varepsilon}(u)=\frac{F_{\varepsilon}(u)-m_{\varepsilon}}{\varepsilon}=\sum_{i \neq j \in \Omega_{\varepsilon}} \varepsilon^{d-1} c_{i, j}^{\varepsilon}\left(1-u_{i} u_{j}\right) .
$$

Copyright (c) by SIAM. Unauthorized reproduction of this article is prohibited. 
We identify the functions $u$ with their piecewise constant interpolations on the cells of the lattice $\varepsilon \mathbb{Z}^{d}$. Note that, with this identification, if we split $E_{\varepsilon}$ into the contribution accounting for the interactions between nearest neighbors and between all the other pairs, we may rewrite it as

$$
E_{\varepsilon}(u)=\int_{S_{u}} a_{\varepsilon}(x) d \mathcal{H}^{d-1}+\int_{\Omega} \int_{\Omega} K_{\varepsilon}(x, y)|u(x)-u(y)| d x d y+O(1)
$$

for suitable functions $a_{\varepsilon}(\cdot)$ and $K_{\varepsilon}(\cdot, \cdot)$, where the additional $O(1)$ term is due to the energetic contribution of the interactions near $\partial \Omega$.

The asymptotic behavior as $\varepsilon \rightarrow 0$ of the energies $E_{\varepsilon}$ can be described by using the methods of $\Gamma$-convergence (see $[13,18]$ ). We make assumptions of two types on the constants $c_{i, j}^{\varepsilon}$ : a coerciveness hypothesis on nearest neighbors, namely, that $c_{i, j}^{\varepsilon} \geq$ $c>0$ when $|j-1|=1$, which ensures that the limit is finite only on $B V(\Omega ;\{ \pm 1\})$, and a decay assumption as $|j-i| \rightarrow+\infty$, that allows us to neglect very-long-range interactions. We point out that in this case the nonlocal term on the right-hand side of (1.5) gives only a contribution, as $\varepsilon \rightarrow 0$, to the surface energy. Indeed, under these conditions we show that, up to passing to a subsequence, the functionals $E_{\varepsilon}$ $\Gamma$-converge to an integral surface functional defined on $B V(\Omega ;\{ \pm 1\})$ and of the form

$$
\int_{S_{u}} \varphi\left(x, \nu_{u}\right) d \mathcal{H}^{d-1}
$$

(see Theorem 4.2).

To perform our analysis, we apply an abstract method, originally exploited in the homogenization theory for multiple integrals and then adapted to a discrete setting in the continuum approximation, by $\Gamma$-convergence, of discrete models in nonlinear elasticity (see $[4,6])$. It amounts to applying a localization argument which allows us to regard our functionals and their $\Gamma$-limits as defined on functions and set and then to prove that all the hypotheses of an integral representation result of [12] (see Theorem 2.2) are fulfilled. We point out that, in applying this method, the surface scaling of the energies considered here requires in some steps a finer analysis than that developed in the bulk case considered in [4] and [6] (see in particular the proof of Proposition 4.5).

In the case of periodic interactions, corresponding to having $c_{i, j}^{\varepsilon}=c(i, j-i)$ with $c(\cdot, \xi)[0, k)^{d}$-periodic for some $k \in \mathbb{N}$ and for any $\xi \in \mathbb{Z}^{d}$, we may derive by our integral representation result an alternative proof of the homogenization result proved in [15]. Indeed, in this case the whole family of functionals $E_{\varepsilon} \Gamma$-converges to a homogenized surface energy of the form

$$
\int_{S_{u}} \varphi_{h o m}\left(\nu_{u}\right) d \mathcal{H}^{d-1}
$$

with $\varphi_{\text {hom }}$ defined by a suitable asymptotic formula (see Theorem 4.7).

As a consequence of our $\Gamma$-convergence results, in section 4.2 we derive the convergence of minimum problems involving discrete energies as in (1.4) to the corresponding minimum problems involving the limiting energy. We focus on boundary value problems and minimum problems with a prescribed volume fraction.

We underline that we limit our analysis to square lattices, but our results can be easily extended to any Bravais lattice or multilattice (see also [7] for an extension of these results to stochastic lattices). Moreover, we provide an extension of our analysis 
to the case of energies accounting for multibody interactions of the form, for a given $M \in \mathbb{N}$,

$$
E_{\varepsilon}^{M}(u)=\sum_{i_{1}, \ldots, i_{M} \in \Omega_{\varepsilon}} \varepsilon^{d-1} \psi_{\varepsilon}\left(i_{1}, \ldots, i_{M}, u_{i_{1}}, \ldots, u_{i_{M}}\right)
$$

Here the ferromagnetic behavior of $E_{\varepsilon}^{M}$ is ensured by the assumption that the potentials $\psi_{\varepsilon}$ are nonnegative and equal 0 only on the uniform configurations $u_{1}=u_{2}=$ $\cdots=u_{M}$. Under coercivity and decay assumptions analogous to those given in the case of pairwise interactions and applying the same argument exploited in that case, it is possible to show that the same integral representation and homogenization results hold for the $\Gamma$-limits of energies of the form (1.8) (see Theorems 4.13 and 4.14). Moreover, we provide an example of energies accounting for nearest-neighbor 3-body interactions, obtained by adding a term in the potentials of the energies in (1.1) which penalizes changes of direction in the interfaces. The interesting effect here is that such an additional term does not give any contribution to the limiting energy, but acts as a selector of the ground states of $E_{\varepsilon}$ (see Example 4.15).

Our decay assumptions on the constants $c_{i, j}^{\varepsilon}$ are somehow the most general to guarantee the locality of the continuum limit. Indeed, in section 5 we show that, if they are violated, we can approximate nonlocal functionals of the form

$$
\int_{S_{u}} \varphi\left(x, \nu_{u}\right) d \mathcal{H}^{d-1}+\int_{\Omega} \int_{\Omega} K(x, y) g(u(x), u(y)) d x d y,
$$

which resembles the representation of $E_{\varepsilon}$ in (1.5). We focus on the approximation of two relevant examples: fractional perimeters and Ohta-Kawasaki-type energies. The first ones correspond to a nonlocal term on the right-hand side of (1.9) of the form

$$
\int_{\Omega} \int_{\Omega} \frac{|u(y)-u(x)|}{|y-x|^{d+s}} d x d y
$$

for $s \in(0,1)$, which is one quarter of the fractional Sobolev space seminorm $\frac{1}{4}|u|_{W^{s, 1}(\Omega)}$. The second ones correspond to a nonlocal term of the form

$$
\gamma_{0} \int_{\Omega} \int_{\Omega} G(x, y) u(x) u(y) d x d y
$$

where $\gamma_{0}>0$ and $G$ is the Green's function of the Laplacian with Neumann boundary conditions on $\partial \Omega$. The original model proposed by Ohta-Kawasaki in [23] in the studies of energy-driven pattern forming systems is based on energies of the form

$$
\mathcal{O} \mathcal{K}_{\varepsilon}(u)=\varepsilon \int_{\Omega}|\nabla u|^{2} d x+\frac{1}{\varepsilon} \int_{\Omega}\left(1-u^{2}\right)^{2} d x+\gamma_{0} \int_{\Omega} \int_{\Omega} G(x, y) u(x) u(y) d x d y
$$

and in the analysis of their minima subject to a prescribed volume fraction. The first two integrals in (1.10) form the so called "Modica-Mortola" energy which $\Gamma$ converges as $\varepsilon \rightarrow 0$ to the perimeter functional (see [19]). It can be easily shown that the nonlocal term in (5.9) is an $L^{1}$ continuous perturbation of the Modica-Mortola energy. As a consequence, the functionals $\mathcal{O K}_{\varepsilon} \Gamma$-converge as $\varepsilon \rightarrow 0$ with respect to the $L^{1}(\Omega)$ norm to the functional finite on $B V(\Omega ;\{ \pm 1\})$ and defined by

$$
\mathcal{O K}(u):=\frac{8}{3} \mathcal{H}^{d-1}\left(S_{u}\right)+\gamma_{0} \int_{\Omega} \int_{\Omega} G(x, y) u(x) u(y) d x d y .
$$

Copyright (C) by SIAM. Unauthorized reproduction of this article is prohibited. 
We provide a variational approximation of an anisotropic version of the functional in (1.11), given by

$$
\int_{S_{u}}\left|\nu_{u}\right|_{1} d \mathcal{H}^{d-1}\left(S_{u}\right)+\gamma_{0} \int_{\Omega} \int_{\Omega} G(x, y) u(x) u(y) d x d y .
$$

The idea is that the first term can be approximated by the discrete functionals in (1.1), while the nonlocal term is approximated just by "discretizing" the double integral. Hence we show that the functional in (1.12) is the $\Gamma$-limit of the sequence of functionals defined by

$$
\hat{E}_{\varepsilon}(u)=-\sum_{|j-i|=1} \varepsilon^{d} u_{i} u_{j}+\gamma_{0} \sum_{i, j \in \Omega_{\varepsilon}} \varepsilon^{2 d+1} G(\varepsilon i, \varepsilon j) u_{j} u_{i} .
$$

The result can be also extended to the periodic case, when $\Omega=\mathbb{T}^{d}$, and to functions satisfying a mean constraint. By virtue of a result proved in [1] and thanks to our approximation, we deduce that lamellar configurations are nearby local minimizers of $\hat{E}_{\varepsilon}$ (see Theorem 5.9). We point out that the periodicity of minimizers of energies for spin systems with competing interactions and different scalings, which resembles the behavior of Ohta-Kawasaki-type energies, has been investigated in many papers (see, for instance, $[21,22])$. It would be interesting, in our opinion, to recast the analysis of those models in our framework.

The last question we address is, in the case there is a competition between ferromagnetic and antiferromagnetic interactions, that is, no constraint on the sign of the constants $c_{i, j}^{\varepsilon}$ is assumed, whether energies of type (1.3) still have a ferromagnetic behavior or, equivalently, whether the ground states are still the uniform states $u \equiv 1$ and $u \equiv-1$ and the continuum limit of the scaled energies is an interfacial energy of the form (1.6). We restrict our analysis to the homogeneous and "short-range" case, that is, $c_{i, j}^{\varepsilon}=c_{j-i}$ for all $i$ and $j$ and $c_{\xi}=0$ if $|\xi|>R$, for some $R>0$. We show that, given $M \in \mathbb{N}$, with $M \geq R$, and setting $C_{M}=[0, M]^{d} \cap \mathbb{Z}^{d}$, the energies in (1.3) can be rewritten as

$$
F_{\varepsilon}(u)=\sum_{i \in \Omega_{\varepsilon}} \varepsilon^{d} F_{c e l l}\left(u, i+C_{M}\right)+O(\varepsilon)
$$

for a suitable function $F_{\text {cell }}$ accounting for the interactions within the cells $i+C_{M}$. Then the condition which ensures ferromagnetic behavior is that $F_{\text {cell }}$ is minimized only by the uniform states 1 and -1 (see Definition 6.3). Under this condition we study the $\Gamma$-limit of the scaled energies $E_{\varepsilon}$ defined as in (1.4). Since it is not our purpose in this paper to investigate boundary layer effects, we limit our analysis to the case in which $\Omega$ is a torus. Thus, by applying blow-up techniques, we show that the $\Gamma$-limit of $E_{\varepsilon}$ still has an integral representation of the form (1.7) with $\varphi$ defined by a suitable asymptotic formula (see Theorem 6.5). Eventually, we provide some one dimensional examples to better explain our analysis and to show its applicability.

2. Notation and preliminary results. In what follows, given $x, y \in \mathbb{R}^{d}$ we denote by $(x, y)$ the usual scalar product in $\mathbb{R}^{d}$ and we set $|x|=\sqrt{(x, x)}$. Moreover we denote by $|\cdot|_{1}$ the $\ell^{1}$-norm in $\mathbb{R}^{d}$ defined as $|x|_{1}=\left|x_{1}\right|+\cdots+\left|x_{d}\right|$. Given $t>0$, we will denote by $[t]$ the integer part of $t$. For any measurable $E \subset \mathbb{R}^{d}$ we denote by $|E|$ the $d$-dimensional Lebesgue measure of $E$ and by $\mathcal{H}^{d-1}(E)$ its $(d-1)$-dimensional Hausdorff measure. Given $x_{0} \in \mathbb{R}^{d}, \nu \in S^{d-1}$, and $\rho>0$, we denote by $Q_{\nu}\left(x_{0}, \rho\right)$ any cube centered in $x_{0}$, with side length $\rho$ and one face orthogonal to $\nu$. We underline 
that, even if the choice of $Q_{\nu}\left(x_{0}, \rho\right)$ is not unique, the results we will present in the following are independent of such a choice. We drop the dependence on $\nu, x_{0}$, or $\rho$ whenever $\nu=e_{i}$ for $i \in\{1,2, \ldots, d\}, x_{0}=0$ or $\rho=1$, respectively, and we set $Q:=\left(-\frac{1}{2}, \frac{1}{2}\right)^{d}$.

Next we recall some basic properties of functions of bounded variation with values in $\{-1,1\}$ (see $[8,11]$ for a general exposition of the subject). Let $A$ be an open subset of $\mathbb{R}^{d}$. We denote by $B V(A ;\{ \pm 1\})$ the set of measurable functions $u: A \rightarrow\{-1,1\}$ whose distributional derivative $D u$ is a measure with bounded total variation. We denote by $S(u)$ the jump set of $u$ and by $\nu_{u}(x)$ the measure theoretic inner normal to $S(u)$ at $x$, which is defined for $\mathcal{H}^{d-1}$ a.e. $x \in S(u)$. Note that if $u \in B V(A ;\{ \pm 1\})$, then $E:=\{x \in A: u(x)=1\}$ is a set of finite perimeter in $A$ and $\mathcal{H}^{d-1}\left(S_{u}\right)=$ $\operatorname{Per}(E, A)$, where $\operatorname{Per}(E, A)$ denotes the euclidean perimeter of $E$ in $A$.

Eventually, for any measurable set $E$ and for any positive real or vector measure $\mu$, we write $\mu\llcorner E$ to denote the restriction of the measure $\mu$ to $E$ defined as $\mu\llcorner E(A):=$ $\mu(E \cap A)$ for any measurable set $A$.

For the reader's convenience we recall the following compactness result (see $[8$, 11]).

Theorem 2.1. Let $u_{k} \in B V(A ;\{ \pm 1\})$ such that

$$
\sup _{k} \mathcal{H}^{d-1}\left(S\left(u_{k}\right)\right)<+\infty .
$$

Then there exists a subsequence (not relabeled) and $u \in B V(A ;\{ \pm 1\})$ such that $u_{k} \rightarrow$ $u$ in the $L^{1}$ convergence.

A main tool in what follows will be the following representation result obtained in [12]. We state here the result only in the particular case of functionals defined on $B V(\Omega ;\{ \pm 1\}) \times \mathcal{A}(\Omega)$ and satisfying an additional symmetry property, although a more general theorem holds. Let $\Omega$ be a bounded open subset of $\mathbb{R}^{d}$ and denote by $\mathcal{A}(\Omega)$ the family of open subsets of $\Omega$.

Theorem 2.2. Let $\mathcal{F}: B V(\Omega ;\{ \pm 1\}) \times \mathcal{A}(\Omega) \rightarrow[0,+\infty)$ satisfy for every $(u, A) \in$ $B V(\Omega ;\{ \pm 1\}) \times \mathcal{A}(\Omega)$ the following hypotheses:

(i) $\mathcal{F}(u, \cdot)$ is the restriction to $\mathcal{A}(\Omega)$ of a Radon measure;

(ii) $\mathcal{F}(u, A)=\mathcal{F}(v, A)$ whenever $u=v$ a.e. on $A \in \mathcal{A}(\Omega)$;

(iii) $\mathcal{F}(\cdot, A)$ is $L^{1}(\Omega)$ lower semicontinuous;

(iv) there exists $C>0$ such that

$$
\frac{1}{C} \mathcal{H}^{d-1}\left(S_{u} \cap A\right) \leq \mathcal{F}(u, A) \leq C \mathcal{H}^{d-1}\left(S_{u} \cap A\right) ;
$$

(v) $\mathcal{F}(u, A)=\mathcal{F}(-u, A)$.

Then for every $u \in B V(\Omega ;\{ \pm 1\})$ and $A \in \mathcal{A}(\Omega)$

$$
\mathcal{F}(u, A)=\int_{S_{u} \cap A} \varphi\left(x, \nu_{u}\right) d \mathcal{H}^{d-1}
$$

with

$$
\varphi\left(x_{0}, \nu\right)=\limsup _{\rho \rightarrow 0} \frac{m\left(u_{x_{0}, \nu}, Q_{\nu}\left(x_{0}, \rho\right)\right)}{\rho^{d-1}}
$$

where

$$
u_{x_{0}, \nu}(x):= \begin{cases}1 & \text { if }\left\langle x-x_{0}, \nu\right\rangle \geq 0 \\ -1 & \text { otherwise }\end{cases}
$$

Copyright (c) by SIAM. Unauthorized reproduction of this article is prohibited. 
and for any $(v, A) \in B V(\Omega ;\{ \pm 1\}) \times \mathcal{A}(\Omega)$ we set

$$
m(v, A)=\inf \{\mathcal{F}(w, A): w \in B V(A ;\{ \pm 1\}), w=v \text { in a neighborhood of } \partial A\} .
$$

The sets of functions of bounded variation are also defined in a periodic setting: we denote by $\mathbb{T}^{d}$ the d-dimensional flat torus of unit volume, which can be identified with the semi-open unit cube $[0,1)^{d}$, and by $B V\left(\mathbb{T}^{d} ;\{ \pm 1\}\right)$ the set of functions $u \in$ $B V_{l o c}\left(\mathbb{R}^{d} ;\{ \pm 1\}\right)$ which are $[0,1)^{d}$-periodic. Moreover, with a slight abuse of notation, for such functions $u$ we identify $S(u)$ with $S(u) \cap[0,1)^{d}$.

Eventually we recall a result proved by Kohn and Sternberg in [20] on the approximation of local minimizers by $\Gamma$-convergence.

THEOREM 2.3. Let $(X, d)$ be a metric space and let $\left(F_{n}\right)_{n}$ be a sequence of lower semicontinuous and equicoercive functionals from $X$ to $\mathbb{R} \cup\{+\infty\}$. Assume that $\left(F_{n}\right)_{n} \Gamma$-converges to $F$ and let $x$ be an isolated local minimizer of $F$. Then there exist $x_{n} \rightarrow x$ such that $x_{n}$ is a local minimizer of $F_{n}$ for $n$ large enough.

3. Setting. In what follows $\Omega$ will denote a bounded open set of $\mathbb{R}^{d}$ with Lipschitz boundary. For fixed $\varepsilon>0$ we denote by $\mathcal{P} C_{\varepsilon}(\Omega)$ the set of functions

$$
\mathcal{P} C_{\varepsilon}(\Omega):=\left\{u: \varepsilon \mathbb{Z}^{d} \cap \Omega \rightarrow\{-1,1\}\right\} .
$$

In order to carry on our analysis, it is convenient to regard $\mathcal{P} C_{\varepsilon}(\Omega)$ as a subset in $L^{1}(\Omega)$. To this aim we will identify a function $u \in \mathcal{P} C_{\varepsilon}(\Omega)$ with its piecewise-constant interpolation on the $\varepsilon$-cubes centered in the lattice, still denoted by $u$. More precisely, we set $u(z)=0$ if $z \in \varepsilon \mathbb{Z}^{d} \backslash \Omega$ and $u(x)=u\left(z_{x}^{\varepsilon}\right)$, where $z_{x}^{\varepsilon} \in \mathbb{Z}^{d}$ is the closest point to $x$ (which is uniquely defined up to a set of zero measure). Other similar interpolations could be taken into account, actually not affecting our asymptotic analysis.

Thus, with fixed $\varepsilon>0$, we consider energies $F_{\varepsilon}: \mathcal{P} C_{\varepsilon}(\Omega) \rightarrow \mathbb{R}$ of the form

$$
F_{\varepsilon}(u)=-\sum_{i \neq j \in \Omega_{\varepsilon}} c_{i, j}^{\varepsilon} \varepsilon^{d} u_{i} u_{j}
$$

where we use the notation $\Omega_{\varepsilon}:=\mathbb{Z}^{d} \cap\left(\varepsilon^{-1} \Omega\right), u_{i}=u(\varepsilon i)$. Up to the change of variable $\xi=j-i$, setting $c_{i, \xi}^{\varepsilon}=c_{i, i+\xi}^{\varepsilon}$, we may equivalently write $F_{\varepsilon}$ as

$$
F_{\varepsilon}(u)=-\sum_{\xi \in \mathbb{Z}^{d} \backslash\{0\}} \sum_{i, i+\xi \in \Omega_{\varepsilon}} c_{i, \xi}^{\varepsilon} \varepsilon^{d} u_{i} u_{i+\xi}
$$

In what follows we will perform an asymptotic analysis of energies suitably scaled, focusing first on the case of "ferromagnetic-type" pairwise interactions.

4. Ferromagnetic case: integral representation of the continuum limits. In this section we treat the case $c_{i, \xi}^{\varepsilon} \geq 0$ for all $i, \xi$, and $\varepsilon$. Under this hypothesis uniform states are ground states. Setting $m_{\varepsilon}:=\min F_{\varepsilon}(u)=F_{\varepsilon}( \pm 1)$, we then consider the scaled energies

$$
E_{\varepsilon}(u)=\frac{F_{\varepsilon}(u)-m_{\varepsilon}}{\varepsilon}=\sum_{\xi \in \mathbb{Z}^{d}} \sum_{i, i+\xi \in \Omega_{\varepsilon}} c_{i, \xi}^{\varepsilon} \varepsilon^{d-1}\left(1-u_{i} u_{i+\xi}\right) .
$$

Copyright $@$ by SIAM. Unauthorized reproduction of this article is prohibited. 
Note that $E_{\varepsilon}$ can be rewritten as

$$
\begin{aligned}
E_{\varepsilon}(u) & =\frac{1}{2} \sum_{\xi \in \mathbb{Z}^{d}} \sum_{i, i+\xi \in \Omega_{\varepsilon}} c_{i, \xi^{\varepsilon}}^{\varepsilon} \varepsilon^{d-1}\left(u_{i+\xi}-u_{i}\right)^{2} \\
& =\sum_{\xi \in \mathbb{Z}^{d}} \sum_{i, i+\xi \in \Omega_{\varepsilon}} c_{i, \xi^{\varepsilon}}^{\varepsilon} \varepsilon^{d-1}\left|u_{i+\xi}-u_{i}\right| \\
& =\sum_{\xi \in \mathbb{Z}^{d}} \sum_{i, i+\xi \in \Omega_{\varepsilon}} c_{i, \xi}^{\varepsilon}|\xi| \varepsilon^{d} \frac{\left|u_{i+\xi}-u_{i}\right|}{\varepsilon|\xi|}
\end{aligned}
$$

i.e., our energies depend linearly on difference quotients.

We fix the following set of hypotheses on $\left(c_{i, \xi}^{\varepsilon}\right)$ :

(H1) (coerciveness) $c_{i, e_{k}}^{\varepsilon} \geq c>0$ for all $\varepsilon>0, i \in \mathbb{Z}^{d}$, and $k \in\{1, \ldots, d\}$;

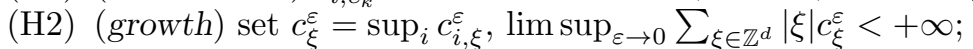

(H3) (decay) for all $\delta>0$ there exists $R_{\delta}>0$ such that $\limsup _{\varepsilon \rightarrow 0} \sum_{|\xi|>R_{\delta}}|\xi| c_{\xi}^{\varepsilon}$ $<\delta$.

With a little abuse of notation, we identify the functionals $E_{\varepsilon}$ by their extension on $L^{1}(\Omega)$ by setting

$$
E_{\varepsilon}(u)= \begin{cases}\sum_{\xi \in \mathbb{Z}^{d}} \sum_{i, i+\xi \in \Omega_{\varepsilon}} c_{i, \xi}^{\varepsilon} \varepsilon^{d-1}\left|u_{i+\xi}-u_{i}\right| & \text { if } u \in \mathcal{P} C_{\varepsilon}(\Omega), \\ +\infty & \text { otherwise in } L^{1}(\Omega) .\end{cases}
$$

We also define a localized version of our energies on regular open sets. Let $\mathcal{A}^{\text {reg }}(\Omega)$ be the subfamily of open subsets of $\Omega$ with Lipschitz boundary. For any $A \in \mathcal{A}^{\text {reg }}(\Omega)$, we isolate the contributions due to interactions within the set $A$ by defining

$$
E_{\varepsilon}(u, A)= \begin{cases}\sum_{\xi \in \mathbb{Z}^{d}} \sum_{i, i+\xi \in A_{\varepsilon}} c_{i, \xi}^{\varepsilon} \varepsilon^{d-1}\left|u_{i+\xi}-u_{i}\right| & \text { if } u \in \mathcal{P} C_{\varepsilon}(\Omega), \\ +\infty & \text { otherwise in } L^{1}(\Omega),\end{cases}
$$

where $A_{\varepsilon}=\mathbb{Z}^{d} \cap\left(\varepsilon^{-1} A\right)$. Moreover we will denote by $E^{\prime}(\cdot, A)$ and $E^{\prime \prime}(\cdot, A)$ the $\Gamma$-liminf $\tilde{\varepsilon}_{\varepsilon \rightarrow 0}$ and the $\Gamma$-lim $\sup _{\varepsilon \rightarrow 0}$ of $E_{\varepsilon}(\cdot, A)$, respectively.

Remark 4.1. We could have also considered a localized version of $E_{\varepsilon}$ on any open set $A \in \mathcal{A}(\Omega)$. In such a case, in order to have an upper bound with a surface energy of type $\mathcal{H}^{d-1}\left(S_{u} \cap A\right)$ (see Proposition (4.4)), we should have slightly modified the definition of $E_{\varepsilon}(\cdot, A)$ accounting only for pairwise contributions indexed by couples $(i, i+\xi)$ such that the whole segment joining $\varepsilon i$ and $\varepsilon(i+\xi)$ lies in $A$. Note that this alternative definition of $E_{\varepsilon}(\cdot, A)$ is asymptotically equivalent to that in (4.3), as $\varepsilon$ tends to 0 , in the case of an open set $A$ with Lipschitz boundary. For the sake of simplicity we prefer to deal with the class $\mathcal{A}^{\text {reg }}(\Omega)$, extending suitably $E^{\prime}(\cdot, A), E^{\prime \prime}(\cdot, A)$ to $\mathcal{A}(\Omega)$ as a last step.

We now state the main result of this section.

TheOrem 4.2. Let $\left(c_{i, \xi}^{\varepsilon}\right)$ satisfy $(\mathrm{H} 1)-(\mathrm{H} 3)$ and let $E_{\varepsilon}$ be defined by (4.2). Then for any sequence $\varepsilon_{n} \rightarrow 0$ there exist a subsequence $\left(\varepsilon_{n_{k}}\right)$ and a function $\varphi: \Omega \times S^{d-1} \rightarrow$ 
$[0,+\infty)$ such that the functionals $E_{\varepsilon_{n_{k}}} \Gamma$-converge with respect to the $L^{1}(\Omega)$ strong topology to the functional $E: L^{1}(\Omega) \rightarrow[0,+\infty]$ defined by

$$
E(u)= \begin{cases}\int_{S_{u}} \varphi\left(x, \nu_{u}\right) d \mathcal{H}^{d-1} & \text { if } u \in B V(\Omega ;\{ \pm 1\}), \\ +\infty & \text { otherwise. }\end{cases}
$$

Moreover for any $u \in B V(\Omega ;\{ \pm 1\})$ and $A \in \mathcal{A}^{\text {reg }}(\Omega)$ there holds

$$
\Gamma-\lim _{k} E_{\varepsilon_{n_{k}}}(u, A)=E(u, A):=\int_{S_{u} \cap A} \varphi\left(x, \nu_{u}\right) d \mathcal{H}^{d-1} .
$$

We postpone the proof of Theorem 4.2, as it will be a consequence of some propositions which show that our limit functionals satisfy all the hypotheses of Theorem 2.2 .

In the next two propositions we show that, thanks to hypotheses (H1) and (H2), for any $A \in \mathcal{A}^{\text {reg }}(\Omega) E^{\prime}(u, A)$ and $E^{\prime \prime}(u, A)$ are finite only on $B V(A ;\{ \pm 1\})$ and satisfy hypothesis (4) of Theorem 2.2 .

Proposition 4.3. Let $\left(c_{i, \xi}^{\varepsilon}\right)$ satisfy (H1) and let $A \in \mathcal{A}^{\text {reg }}(\Omega)$. If $u \in L^{1}(\Omega)$ is such that $E^{\prime}(u, A)$ is finite, then $u \in B V(A ;\{ \pm 1\})$ and

$$
E^{\prime}(u, A) \geq C \mathcal{H}^{d-1}\left(S_{u} \cap A\right)
$$

for some positive constant $C$ independent of $u$ and $A$.

Proof. Let $G_{\varepsilon}$ be the functional accounting only for nearest-neighbor interactions defined by

$$
G_{\varepsilon}(u, A)= \begin{cases}\sum_{k=1}^{d} \sum_{i, i+e_{k} \in A_{\varepsilon}} \varepsilon^{d-1}\left|u_{i+e_{k}}-u_{i}\right| & \text { if } u \in \mathcal{P} C_{\varepsilon}(\Omega) \\ +\infty & \text { otherwise in } L^{1}(\Omega)\end{cases}
$$

In $\left[3\right.$, Theorem 4] it was proved that $G(\cdot, A):=\Gamma-\lim _{\varepsilon \rightarrow 0} G_{\varepsilon}(\cdot, A)$ is finite only on $B V(A ;\{ \pm 1\})$ and

$$
G(u, A)=\int_{S_{u} \cap A}\left|\nu_{u}\right|_{1} d \mathcal{H}^{d-1}, \quad u \in B V(A ;\{ \pm 1\}),
$$

where $|\cdot|_{1}$ is the $\ell^{1}$-norm in $\mathbb{R}^{d}$. Since by $(\mathrm{H} 1)$ we have that $E_{\varepsilon}(\cdot, A) \geq C G_{\varepsilon}(\cdot, A)$, the conclusion easily follows.

Proposition 4.4. Let $\left(c_{i, \xi}^{\varepsilon}\right)$ satisfy $(\mathrm{H} 2)$. Then for every $u \in B V(\Omega ;\{ \pm 1\})$ and for every $A \in \mathcal{A}^{\text {reg }}(\Omega)$ there holds

$$
E^{\prime \prime}(u, A) \leq C \mathcal{H}^{d-1}\left(S_{u} \cap A\right)
$$

for some positive constant $C$ independent of $u$ and $A$.

Proof. Let us fix $A \in \mathcal{A}^{\text {reg }}(\Omega)$. By a density argument, it suffices to prove (4.7) for $u$ such that $S_{u}$ is a polyhedral set. Indeed, by classical theorems (see [11, 17]), for 
every set $E$ of finite perimeter in $\mathbb{R}^{d}$ there exists a sequence of polyhedral sets $\left(E_{k}\right)_{k}$ such that $\lim _{k \rightarrow+\infty}\left|\left(E \Delta E_{k}\right) \cap A\right|=0$ and $\lim _{k \rightarrow+\infty} \operatorname{Per}\left(E_{k}, A\right)=\operatorname{Per}(E, A)$. With fixed $u \in$ $B V(\Omega ;\{ \pm 1\})$, set $E:=\{x \in A: u(x)=1\}$ and let $E_{k}$ be the corresponding sequence of polyhedral sets as above. Then the sequence of functions $u_{k} \in B V(\Omega ;\{ \pm 1\})$ defined as

$$
u_{k}(x)= \begin{cases}\chi_{E_{k}}-\chi_{A \backslash E_{k}} & \text { if } x \in A, \\ u(x) & \text { if } x \in \Omega \backslash A,\end{cases}
$$

converges to $u$ in $L^{1}(\Omega)$ and the lower semicontinuity of $E^{\prime \prime}(\cdot, A)$ yields that inequality (4.7), satisfied by $u_{k}$, passes to the limit as $k \rightarrow+\infty$.

Up to a localization argument we can further reduce to the case when $S_{u}$ is a hyperplane, that is $u=u_{0, \nu}$ defined in (2.2). Thus, having defined $u_{\varepsilon}$ as the pointwise interpolation of $u$, i.e., $\left(u_{\varepsilon}\right)_{i}:=u(\varepsilon i)$, we have that $u_{\varepsilon} \rightarrow u$ strongly in $L^{1}(\Omega)$. Moreover, for any pair $i, i+\xi \in \mathbb{Z}^{d} \cap \varepsilon^{-1} A$ such that $u(\varepsilon i) \neq u(\varepsilon(i+\xi))$, the segment joining $\varepsilon i$ and $\varepsilon(i+\xi)$ crosses the jump set $S_{u}$. Hence, the energetic contribution of $u_{\varepsilon}$ in $A$ can be estimated by

$$
E_{\varepsilon}\left(u_{\varepsilon}, A\right) \leq C \sum_{\xi \in \mathbb{Z}^{d}} \varepsilon^{d-1} c_{\xi}^{\varepsilon} \#\left(A_{\varepsilon}^{\xi} \cap \varepsilon \mathbb{Z}^{d}\right),
$$

where $A_{\varepsilon}^{\xi}:=\left\{x=y+t \xi: y \in S_{u},-\varepsilon \leq t \leq 0\right\} \cap A$. Since, by the regularity of $S_{u}$ and $\partial A$,

$$
\#\left(A_{\varepsilon}^{\xi} \cap \varepsilon \mathbb{Z}^{d}\right) \leq C \varepsilon^{-d}\left|A_{\varepsilon}^{\xi}\right| \leq C \varepsilon^{1-d}|\xi| \mathcal{H}^{d-1}\left(S_{u} \cap A\right),
$$

we eventually get

$$
E_{\varepsilon}\left(u_{\varepsilon}, A\right) \leq C \mathcal{H}^{d-1}\left(S_{u} \cap A\right) \sum_{\xi \in \mathbb{Z}^{d}}|\xi| c_{\xi}^{\varepsilon} .
$$

Passing to the limit as $\varepsilon \rightarrow 0$, by (H2) we get the conclusion.

Proposition 4.5. Let $\left(c_{i, \xi}^{\varepsilon}\right)$ satisfy $(\mathrm{H} 1)-(\mathrm{H} 3)$ and let $A, B, A^{\prime} \in \mathcal{A}^{\text {reg }}(\Omega)$ be given with $A^{\prime} \subset \subset A$. Then for any $u \in B V(\Omega ;\{ \pm 1\})$

$$
E^{\prime \prime}\left(u, A^{\prime} \cup B\right) \leq E^{\prime \prime}(u, A)+E^{\prime \prime}(u, B) .
$$

Proof. It is not restrictive to assume as a further hypothesis that $A^{\prime}$ is an open set with smooth boundary. Indeed, being $E^{\prime \prime}(u, \cdot)$ an increasing set function on $\mathcal{A}^{\text {reg }}(\Omega)$, it is enough to notice that there exists an open set $A^{\prime \prime}$ with smooth boundary such that $A^{\prime} \subset \subset A^{\prime \prime} \subset \subset A$. Such a set can actually be selected by using a convolution and selection of level sets procedure.

Without loss of generality we may also suppose $E^{\prime \prime}(u, A)$ and $E^{\prime \prime}(u, B)$ finite. Let $u_{\varepsilon}, v_{\varepsilon}$ both converge to $u$ in $L^{1}(\Omega)$ and be such that

$$
\limsup _{\varepsilon \rightarrow 0} E_{\varepsilon}\left(u_{\varepsilon}, A\right)=E^{\prime \prime}(u, A), \quad \limsup _{\varepsilon \rightarrow 0} E_{\varepsilon}\left(v_{\varepsilon}, B\right)=E^{\prime \prime}(u, B) .
$$

Fix $\delta>0$ and let $R_{\delta}$ be provided by hypothesis (H3). Since $A^{\prime}$ has a smooth boundary, the distance function $d(x):=\operatorname{dist}\left(x, A^{\prime}\right)$ inherits the same regularity in a suitable neighborhood $U$ of $A^{\prime}$. Set

$$
\bar{d}:=\operatorname{dist}\left(A^{\prime}, U^{c}\right), \quad N_{\varepsilon}:=\left[\frac{\bar{d}}{\varepsilon R_{\delta}}\right] .
$$


For $t \in[0, \bar{d}]$ denote also

$$
A^{t}:=\left\{x \in A: \operatorname{dist}\left(x, A^{\prime}\right) \leq t\right\} .
$$

Plugging the classical Sard theorem in the Fleming-Rishel coarea formula, for $\mathcal{L}^{1}$-a.e. $t \in[0, \bar{d}] A^{t}$ is a set of finite perimeter with smooth boundary. Moreover, for any fixed integer $k \in\left\{1, \ldots, N_{\varepsilon}\right\}$, we can select values $t_{k} \in\left(k \varepsilon R_{\delta},(k+1) \varepsilon R_{\delta}\right)$ such that $A^{t_{k}}$ is a smooth set and satisfies

$$
\begin{aligned}
\varepsilon R_{\delta} \mathcal{H}^{d-1}\left(\partial A^{t_{k}}\right) & \leq \int_{k \varepsilon R_{\delta}}^{(k+1) \varepsilon R_{\delta}} \mathcal{H}^{d-1}\left(\partial^{*} A^{t}\right) d t \\
& \leq \int_{\left\{k \varepsilon R_{\delta} \leq d(x) \leq(k+1) \varepsilon R_{\delta}\right\}}|\nabla d(x)| d x=\left|A^{(k+1) \varepsilon R_{\delta}} \backslash A^{k \varepsilon R_{\delta}}\right| .
\end{aligned}
$$

For any $k \in\left\{1, \ldots, N_{\varepsilon}\right\}$ consider then the family of functions $w_{\varepsilon}^{k}$ still converging to $u$ in $L^{1}(\Omega)$ defined by

$$
w_{\varepsilon}^{k}:=\chi_{A^{t_{k}}} u_{\varepsilon}+\left(1-\chi_{A^{t_{k}}}\right) v_{\varepsilon} .
$$

By taking into account the regularity of $A^{t_{k}}$, we easily compute

$$
\begin{aligned}
E_{\varepsilon}\left(w_{\varepsilon}^{k}, A^{\prime} \cup B\right) \leq E_{\varepsilon}\left(u_{\varepsilon}, A^{t_{k}}\right. & \left.\cap\left(A^{\prime} \cup B\right)\right)+E_{\varepsilon}\left(v_{\varepsilon},\left(A^{t_{k}}\right)^{c} \cap\left(A^{\prime} \cup B\right)\right) \\
& +\sum_{\xi \in \mathbb{Z}^{d}} \sum_{i \in S_{\varepsilon}^{k, \xi}} c_{i, \xi}^{\varepsilon} \varepsilon^{d-1}\left|\left(w_{\varepsilon}^{k}\right)_{i+\xi}-\left(w_{\varepsilon}^{k}\right)_{i}\right| \\
\leq E_{\varepsilon}\left(u_{\varepsilon}, A\right)+ & E_{\varepsilon}\left(v_{\varepsilon}, B\right) \\
& +\sum_{\xi \in \mathbb{Z}^{d}} \sum_{i \in S_{\varepsilon}^{k, \xi}} c_{i, \xi}^{\varepsilon} \varepsilon^{d-1}\left|\left(w_{\varepsilon}^{k}\right)_{i+\xi}-\left(w_{\varepsilon}^{k}\right)_{i}\right|,
\end{aligned}
$$

where $S_{\varepsilon}^{k, \xi}:=\left(\varepsilon^{-1}\left\{x=y+t \xi, y \in \partial A^{t_{k}},-\varepsilon \leq t \leq 0\right\} \cap\left(A^{\prime} \cup B\right)\right) \cap \mathbb{Z}^{d}$. In order to get the desired estimate we will argue by splitting the last term in (4.9) in interactions in $S_{\varepsilon}^{k, \xi}$ with $|\xi|>R_{\delta}$ and with $|\xi| \leq R_{\delta}$, respectively. We will provide different estimates for the two terms.

By arguing as in the proof of Proposition 4.4, thanks to the regularity of $A^{t_{k}}$, we get that

$$
\sum_{|\xi|>R_{\delta}} \sum_{i \in S_{\varepsilon}^{k, \xi}} c_{i, \xi}^{\varepsilon} \varepsilon^{d-1}\left|\left(w_{\varepsilon}^{k}\right)_{i+\xi}-\left(w_{\varepsilon}^{k}\right)_{i}\right| \leq C \mathcal{H}^{d-1}\left(\partial A^{t_{k}}\right) \sum_{|\xi|>R_{\delta}}|\xi| c_{\xi}^{\varepsilon} .
$$

By using the mean property (4.8) of $A^{t_{k}}$ we may refine the inequality above,

$$
\sum_{|\xi|>R_{\delta}} \sum_{i \in S_{\varepsilon}^{k, \xi}} c_{i, \xi}^{\varepsilon} \varepsilon^{d-1}\left|\left(w_{\varepsilon}^{k}\right)_{i+\xi}-\left(w_{\varepsilon}^{k}\right)_{i}\right| \leq C \delta \frac{\left|A^{(k+1) \varepsilon R_{\delta}} \backslash A^{k \varepsilon R_{\delta}}\right|}{\varepsilon R_{\delta}} .
$$

We are left with the estimate of the energy accounting for the interactions in $S_{\varepsilon}^{k, \xi}$ when $|\xi| \leq R_{\delta}$. Note that in this case, by the definition of $A^{k \varepsilon R_{\delta}}$, we have that $S_{\varepsilon}^{k, \xi} \subseteq\left(A^{(k+1) \varepsilon R_{\delta}} \backslash A^{(k-1) \varepsilon R_{\delta}}\right) \cap B \cap \varepsilon \mathbb{Z}^{d}=: S_{\varepsilon}^{k}$. Moreover, we easily get that

$$
\left|\left(w_{\varepsilon}^{k}\right)_{i+\xi}-\left(w_{\varepsilon}^{k}\right)_{i}\right| \leq 2\left(\left|\left(u_{\varepsilon}\right)_{i+\xi}-\left(u_{\varepsilon}\right)_{i}\right|+\left|\left(v_{\varepsilon}\right)_{i+\xi}-\left(v_{\varepsilon}\right)_{i}\right|+\left|\left(v_{\varepsilon}\right)_{i}-\left(u_{\varepsilon}\right)_{i}\right|\right) ;
$$

Copyright (c) by SIAM. Unauthorized reproduction of this article is prohibited. 
hence,

$$
\begin{aligned}
& \sum_{|\xi| \leq R_{\delta}} \sum_{i \in S_{\varepsilon}^{k, \xi}} c_{i, \xi}^{\varepsilon} \varepsilon^{d-1}\left|\left(w_{\varepsilon}^{k}\right)_{i+\xi}-\left(w_{\varepsilon}^{k}\right)_{i}\right| \\
& \leq C\left(E_{\varepsilon}\left(u_{\varepsilon}, S_{\varepsilon}^{k}\right)+E_{\varepsilon}\left(v_{\varepsilon}, S_{\varepsilon}^{k}\right)\right)+C_{\delta} \sum_{i \in S_{\varepsilon}^{k}} \varepsilon^{d-1}\left|\left(v_{\varepsilon}\right)_{i}-\left(u_{\varepsilon}\right)_{i}\right|
\end{aligned}
$$

for some constant $C_{\delta}$ depending only on $R_{\delta}$.

Therefore, summing over $k \in\left\{1, \ldots, N_{\varepsilon}-1\right\}$ and averaging, by (4.9), (4.10), and (4.11), we get

$$
\begin{aligned}
I_{\varepsilon} & :=\frac{1}{N_{\varepsilon}-1} \sum_{k=1}^{N_{\varepsilon}-1}\left(E_{\varepsilon}\left(w_{\varepsilon}^{k}, A^{\prime} \cup B\right)\right) \leq E_{\varepsilon}\left(u_{\varepsilon}, A\right)+E_{\varepsilon}\left(v_{\varepsilon}, B\right) \\
& +\frac{2}{N_{\varepsilon}-1}\left(E_{\varepsilon}\left(u_{\varepsilon}, A \cap B\right)+E_{\varepsilon}\left(v_{\varepsilon}, A \cap B\right)\right)+C_{\delta}^{\prime} \sum_{i \in \Omega_{\varepsilon}} \varepsilon^{d}\left|\left(v_{\varepsilon}\right)_{i}-\left(u_{\varepsilon}\right)_{i}\right|+C \delta\left|A \backslash A^{\prime}\right|
\end{aligned}
$$

$$
\leq E_{\varepsilon}\left(u_{\varepsilon}, A\right)+E_{\varepsilon}\left(v_{\varepsilon}, B\right)+\frac{C}{N_{\varepsilon}}+C \delta+C_{\delta}\left\|v_{\varepsilon}-u_{\varepsilon}\right\|_{L^{1}(\Omega)},
$$

where we have also used that $\cup_{k=0}^{N_{\varepsilon}} A^{(k+1) \varepsilon R_{\delta}} \backslash A^{k \varepsilon R_{\delta}}=\bar{U} \backslash A^{\prime} \subseteq A \backslash A^{\prime}$.

Eventually, for any $\varepsilon>0$ let $k_{\varepsilon} \in\left\{1, \ldots, N_{\varepsilon}-1\right\}$ be such that

$$
E_{\varepsilon}\left(w_{\varepsilon}^{k_{\varepsilon}}, A^{\prime} \cup B\right) \leq I_{\varepsilon} .
$$

Setting $w_{\varepsilon}:=w_{\varepsilon}^{k_{\varepsilon}}$, we have that $w_{\varepsilon} \rightarrow u$ strongly in $L^{1}(\Omega)$ and, by (4.12), (4.13), passing to the limit as $\varepsilon \rightarrow 0$ we infer also that

$$
E^{\prime \prime}\left(u, A^{\prime} \cup B\right) \leq \limsup _{\varepsilon \rightarrow 0} E_{\varepsilon}\left(w_{\varepsilon}, A^{\prime} \cup B\right) \leq E^{\prime \prime}(u, A)+E^{\prime \prime}(v, B)+C \delta .
$$

The conclusion follows by the arbitrariness of $\delta>0$.

In the following proposition we show that $E^{\prime \prime}(\cdot, \cdot)$ satisfies hypothesis (ii) of Theorem 2.2.

Proposition 4.6. Let $\left(c_{i, \xi}^{\varepsilon}\right)$ satisfy $(\mathrm{H} 1)-(\mathrm{H} 3)$. Then, for any $A \in \mathcal{A}^{\text {reg }}(\Omega)$ and for any $u, v \in B V(\Omega ;\{ \pm 1\})$ such that $u=v$ a.e. in $A$, there holds

$$
E^{\prime \prime}(u, A)=E^{\prime \prime}(v, A)
$$

Proof. Let $A \in \mathcal{A}^{\text {reg }}(\Omega)$ and $u, v \in B V(\Omega ;\{ \pm 1\})$ be fixed with $u=v$ a.e. in $A$. Up to reversing the role of $u$ and $v$, it is enough to prove that

$$
E^{\prime \prime}(v, A) \leq E^{\prime \prime}(u, A)
$$

With $\delta>0$ fixed, let $A_{\delta} \in \mathcal{A}^{\text {reg }}(\Omega)$ such that $A_{\delta} \subset \subset A$ and

$$
\mathcal{H}^{d-1}\left\llcorner S_{u}\left(A \backslash \overline{A_{\delta}}\right)<\delta .\right.
$$

Note that $A \backslash \overline{A_{\delta}} \in \mathcal{A}^{\text {reg }}(\Omega)$ and by Proposition 4.4, there holds

$$
E^{\prime \prime}\left(v, A \backslash \overline{A_{\delta}}\right) \leq C \mathcal{H}^{d-1}\left\llcorner S_{u}\left(A \backslash \overline{A_{\delta}}\right) \leq C \delta .\right.
$$

Copyright (c) by SIAM. Unauthorized reproduction of this article is prohibited. 
Let $u_{\varepsilon}$ and $v_{\varepsilon}$ be converging to $u$ and $v$ in $L^{1}(\Omega)$, respectively, and such that

$$
\begin{aligned}
\limsup _{\varepsilon \rightarrow 0} E_{\varepsilon}\left(u_{\varepsilon}, A\right) & =E^{\prime \prime}(u, A), \\
\limsup _{\varepsilon \rightarrow 0} E_{\varepsilon}\left(v_{\varepsilon}, A \backslash \overline{A_{\delta}}\right) & =E^{\prime \prime}\left(v, A \backslash \overline{A_{\delta}}\right) .
\end{aligned}
$$

Arguing as in the proof of Proposition 4.5, with $A^{\prime}=A_{\delta}, B=A \backslash \overline{A_{\delta}}$, we may choose for any $\varepsilon>0$ a set $A^{t_{k_{\varepsilon}}}$ and the relative interpolated function

$$
w_{\varepsilon}^{k_{\varepsilon}}:=\chi_{A^{t_{\varepsilon}}} u_{\varepsilon}+\left(1-\chi_{A^{t_{k_{\varepsilon}}}}\right) v_{\varepsilon}
$$

such that $w_{\varepsilon}^{k_{\varepsilon}}$ converges to $v$ in $L^{1}(\Omega)$ and, by passing to the limsup as $\varepsilon \rightarrow 0$, it holds

$$
E^{\prime \prime}(v, A) \leq \limsup _{\varepsilon \rightarrow 0} E_{\varepsilon}\left(w_{\varepsilon}^{k_{\varepsilon}}, A\right) \leq E^{\prime \prime}(u, A)+E^{\prime \prime}\left(v, A \backslash \overline{A_{\delta}}\right)+C \delta .
$$

Hence (4.14) follows by (4.15) and the arbitrariness of $\delta>0$.

Proof of Theorem 4.2. Taking into account Propositions 4.4 and 4.5, one can infer that for any $u \in B V(\Omega ;\{ \pm 1\}), E^{\prime \prime}(u, \cdot)$ is inner regular and subadditive on $\mathcal{A}^{\text {reg }}(\Omega)$ (see as a reference for this procedure [14, Proposition 11.6]). By the compactness property of the $\Gamma$-convergence and arguing as in [14, Theorem 10.3], we may construct a subsequence $\left(\varepsilon_{n_{k}}\right)$ such that, for any $(u, A) \in B V(\Omega ;\{ \pm 1\}) \times \mathcal{A}^{\text {reg }}(\Omega)$ there exists

$$
\Gamma-\lim _{k} E_{\varepsilon_{n_{k}}}(u, A)=: E(u, A) .
$$

Moreover by setting $E(u, A):=\sup \left\{E\left(u, A^{\prime}\right): A^{\prime} \in \mathcal{A}^{\text {reg }}(\Omega), A^{\prime} \subset A\right\}$ we can extend $E(u, \cdot)$ to $\mathcal{A}(\Omega)$ and easily verify that all the properties enjoyed by $E^{\prime \prime}(\cdot, \cdot)$ stated in Propositions 4.3, 4.4, 4.5, and 4.6 still hold true for $E(\cdot, \cdot)$. Moreover, by Proposition 4.3 ,

$$
\Gamma-\lim _{k} E_{\varepsilon_{n_{k}}}(u)=+\infty
$$

for $u \in L^{1}(\Omega) \backslash B V(\Omega ;\{ \pm 1\})$. So far, it suffices to check that, for every $(u, A) \in$ $B V(\Omega ;\{ \pm 1\}) \times \mathcal{A}(\Omega), E(u, A)$ satisfies all the hypotheses of Theorem 2.2. In fact, the superadditivity property of $E_{\varepsilon}(u, \cdot)$ is preserved in the limit. Thus, thanks to the De Giorgi-Letta criterion (see [14, Theorem 5.2]), hypotheses (i), (ii), and (iv) hold true. By the lower semicontinuity property of the $\Gamma$-limit, hypothesis (iii) is also fulfilled and finally hypothesis $(\mathrm{v})$ holds since it is satisfied by $E_{\varepsilon}(\cdot, A)$ for any $\varepsilon>0$.

The previous argument actually also provides a proof of (4.5).

4.1. Homogenization. Theorem 4.2 allows us to give an alternative proof of the homogenization result proved in [15] when the interaction potentials are periodic in the independent variable. More precisely, assume that $c_{i, \xi}^{\varepsilon}=c_{\xi}(i)$, where, for any $\xi \in \mathbb{Z}^{d}, c_{\xi}: \mathbb{Z}^{d} \rightarrow[0,+\infty)$ is $[0, l]^{d}$-periodic for some $l \in \mathbb{N}$. In this case hypotheses (H1)-(H3) reduce to

$\left(\mathrm{H} 1^{\prime}\right) c_{e_{k}}(i) \geq c>0$ for all $i \in\{0, \ldots, l-1\}^{d}$ and $k \in\{1, \ldots, d\}$;

$\left(\mathrm{H} 2^{\prime}\right) \sum_{\xi \in \mathbb{Z}^{d}}|\xi| c_{\xi}(i)<+\infty$ for all $i \in\{0, \ldots, l-1\}^{d}$. Then the following theorem holds (see [15, Theorem 2.4 and Remark 2.5]).

Theorem 4.7 (homogenization). Let $E_{\varepsilon}$ be defined by (4.2) and let $c_{i, \xi}^{\varepsilon}=c_{\xi}(i)$, where, for any $\xi \in \mathbb{Z}^{d}, c_{\xi}: \mathbb{Z}^{d} \rightarrow[0,+\infty)$ is $[0, l]^{d}$-periodic for some $l \in \mathbb{N}$. If $\left(\mathrm{H} 1^{\prime}\right)$ and $\left(\mathrm{H} 2^{\prime}\right)$ hold, then the functionals $E_{\varepsilon} \Gamma$-converge with respect to the $L^{1}(\Omega)$ strong 
topology, as $\varepsilon \rightarrow 0$, to the homogenized functional $E_{h o m}: L^{1}(\Omega) \rightarrow[0,+\infty]$ defined by

$$
E_{\text {hom }}(u)= \begin{cases}\int_{S_{u}} \varphi_{\text {hom }}\left(\nu_{u}\right) d \mathcal{H}^{d-1} & \text { if } u \in B V(\Omega ;\{ \pm 1\}), \\ +\infty & \text { otherwise, }\end{cases}
$$

where

$$
\begin{array}{r}
\varphi_{\text {hom }}(\nu)=\lim _{T \rightarrow+\infty} T^{1-d} \inf \left\{\sum_{\xi \in \mathbb{Z}^{d}} \sum_{i \in \mathbb{Z}^{d} \cap T Q_{\nu}} c_{\xi}(i) u_{i} u_{i+\xi}: u \in \mathcal{P} C_{1}\left(\mathbb{R}^{d}\right),\right. \\
\left.u(i)=u_{0, \nu}(i) \text { for } i \notin T Q_{\nu}\right\} .
\end{array}
$$

Sketch of the proof. The proof strongly relies upon some arguments and techniques well established in homogenization theory, suitably combined with the results of Theorem 4.2 of the previous section. Here we highlight only its main steps for the reader's convenience.

First of all, by Theorem 4.2, given a sequence $\varepsilon_{n} \rightarrow 0$, we can extract a subsequence $\varepsilon_{n_{k}}$ such that the functionals $E_{\varepsilon_{n_{k}}} \Gamma$-converge to a functional $E$ defined as in (4.4) and for any $u \in B V(\Omega ;\{ \pm 1\})$ and $A \in \mathcal{A}^{r e g}(\Omega)$ there holds

$$
\Gamma-\lim _{k} E_{\varepsilon_{n_{k}}}(u, A)=\int_{S_{u} \cap A} \varphi\left(x, \nu_{u}\right) \mathcal{H}^{d-1} .
$$

Hence, the theorem is proved if we show that $\varphi$ does not depend on $x$ and $\varphi \equiv \varphi_{\text {hom }}$. In order to establish the first claim, by using the periodicity assumption, one easily shows that if we set $E(u, A)=\int_{S_{u} \cap A} \varphi\left(x, \nu_{u}\right) \mathcal{H}^{d-1}$, then

$$
E\left(u_{x, \nu}, B(x, \rho)\right)=E\left(u_{z, \nu}, B(z, \rho)\right)
$$

for any $\nu \in S^{d-1}, x, z \in \Omega$ and $\rho>0$, such that $B(x, \rho) \cup B(z, \rho) \subseteq \Omega$. Thus, the independence of $\varphi$ on $x$ follows from (4.17).

So far, assuming for the sake of simplicity that $0 \in \Omega$, by using the characterization of $\varphi$ in (2.1), we have

$$
\varphi(\nu)=\limsup _{\rho \rightarrow 0} \frac{m\left(u_{0, \nu}, \rho Q_{\nu}\right)}{\rho^{d-1}},
$$

where for any $(v, A) \in B V(\Omega ;\{ \pm 1\}) \times \mathcal{A}(\Omega)$

$$
m(v, A)=\inf \{E(w, A): w \in B V(A ;\{ \pm 1\}), w=v \text { in a neighborhood of } \partial A\} .
$$

Taking into account the convergence of boundary value problems stated in Theorem 4.9 of the subsequent section, applied to $m(v, A)$, together with Remark 4.10, we infer the validity of the cell formula

$$
\begin{array}{r}
\varphi(\nu)=\lim _{k \rightarrow+\infty} T_{k}^{1-d} \inf \left\{\sum_{\underline{\xi} \in \mathbb{Z}^{d(M-1)}} \sum_{i \in \mathbb{Z}^{d} \cap T_{k} Q_{\nu}} c_{\xi}(i) u_{i} u_{i+\xi}: u \in \mathcal{P} C_{1}\left(\mathbb{R}^{d}\right),\right. \\
\left.u(i)=u_{0, \nu}(i) \text { for } i \notin T_{k} Q_{\nu}\right\}
\end{array}
$$

Copyright $\odot$ by SIAM. Unauthorized reproduction of this article is prohibited. 
for a suitable sequence $T_{k} \rightarrow+\infty$. Using the periodicity assumption and hypotheses (H1'), (H2') and following a standard procedure in homogenization theory (we refer for instance to the proof of [4, Proposition 4.2] in the context of discrete models in nonlinear elasticity), it can be shown that the limit in (4.16) exists and is finite for any $\nu \in S^{d-1}$. Hence, by (4.18), we conclude that $\varphi \equiv \varphi_{\text {hom }}$.

As a straightforward application of Theorem 4.2, in the following example we show that if the constants $c_{i, \xi}^{\varepsilon}$ do not satisfy the periodicity assumptions stated above, we may obtain in the continuum limit more general inhomogeneous surface functionals.

Example 4.8. Let $\Omega=(-1,1)^{2}$ and let $c:[-1,1]^{2} \rightarrow \mathbb{R}$ be defined as

$$
c(x)= \begin{cases}1 & \text { if } x \in[0,1] \times[-1,1], \\ 2 & \text { otherwise in }[-1,1]^{2} .\end{cases}
$$

Let $E_{\varepsilon}: L^{1}(\Omega) \rightarrow[0,+\infty]$ be defined as in (4.2) with $d=2, c_{i, \xi}^{\varepsilon}=0$ if $\xi \notin\left\{e_{1}, e_{2}\right\}$, and $c_{i, e_{1}}^{\varepsilon}=c_{i, e_{2}}^{\varepsilon}=c(\varepsilon i)$. Then the functionals $E_{\varepsilon} \Gamma$-converge with respect to the $L^{1}(\Omega)$ strong topology to the functional $E(u)$ defined as in (4.4) with $\varphi(x, \nu)=|\nu|_{1}$ if $x \in$ $[0,1) \times(-1,1)$ and $\varphi(x, \nu)=2|\nu|_{1}$ if $x \in(-1,0) \times(-1,1)$. Indeed, by Theorem 4.2 , it suffices to show that $\Gamma-\lim _{\varepsilon \rightarrow 0} E_{\varepsilon}\left(u_{x_{0}, \nu}\right)=E\left(u_{x_{0}, \nu}\right)$ for every $\left(x_{0}, \nu\right) \in \Omega \times S^{d-1}$. The $\Gamma$-liminf inequality follows by a slicing argument, while, for the $\Gamma$-lim sup inequality, a recovery sequence for $u_{x_{0}, \nu}$ is provided by an infinitesimal right translation of the pointwise interpolation of $u_{x_{0}, \nu}$, defined by $\left(u_{\varepsilon}\right)_{i}=u_{x_{0}, \nu}\left(\varepsilon i-\varepsilon e_{1}\right)$.

More generally, an analogous convergence result could be inferred in any dimension, considering a finite collection of disjoint sets $\left\{A_{k}\right\}_{k=1}^{N}$ in $\Omega$ with $A_{k} \in \mathcal{A}^{r e g}(\Omega)$ and positive constants $c_{1}, \ldots, c_{N}$. Indeed, set $c(x)=\sum_{k=1}^{N} c_{k} \chi_{A_{k}}(x)$ and let $E_{\varepsilon}$ be defined as in (4.2) with $c_{i, \xi}^{\varepsilon}=0$ if $\xi \notin\left\{e_{1}, \ldots, e_{d}\right\}$ and $c_{i, e_{1}}^{\varepsilon}=\cdots=c_{i, e_{d}}^{\varepsilon}=c(\varepsilon i)$. Then as a consequence of Theorem 4.2 and applying the argument above, we could deduce that the functionals $E_{\varepsilon} \Gamma$-converge with respect to the $L^{1}(\Omega)$ strong topology to the functional $E(u)$ defined as in (4.4) with $\varphi(x, \nu)=a(x)|\nu|_{1}$ and $a: \Omega \rightarrow(0,+\infty)$ defined by $a(x)=\sum_{k=1}^{N} c_{k} \chi_{A_{k}}(x)+\sum_{k, h=1}^{N} c_{k} \wedge c_{h} \chi_{\partial A_{k} \cap \partial A_{h}}(x)$. Moreover the same result holds if we replace the piecewise-constant function $c(\cdot)$ with the sequence of continuous functions $c_{\varepsilon}(\cdot):=c * \rho_{\varepsilon}(\cdot)$, where $\rho_{\varepsilon}$ is a convolution kernel supported in the ball $\left\{x \in \mathbb{R}^{d}:|x| \leq \varepsilon\right\}$. Hence, the continuity of $\varphi(\cdot, \nu)$ cannot be inferred by the regularity of $c_{\varepsilon}$.

4.2. Convergence of minimum problems. In this section we established the convergence of minimum problems involving discrete energies as in (4.2) to the corresponding minimum problem involving the limiting energy. We focus first on boundary value problems and afterwards we also treat the case of minimum problems with prescribed volume fraction.

In order to state boundary value problems, let $A \in \mathcal{A}^{\text {reg }}(\Omega)$ and assume $u_{0} \in$ $B V_{\text {loc }}\left(\mathbb{R}^{d} ;\{ \pm 1\}\right)$ to be fixed with $S_{u_{0}}$ polyhedral and transverse to $A$, that is $\mathcal{H}^{d-1}\left(S_{u_{0}} \cap \partial A\right)=0$. Actually, more general boundary data could also be taken into account. Here we restrict our analysis for a boundary datum $u_{0}$ as above just for the sake of simplicity.

For any $\varepsilon, \eta>0$ set

$$
\begin{gathered}
A^{\eta}:=\{x \in A: \operatorname{dist}(x, \partial A)<\eta\}, \\
\mathcal{P} C_{\varepsilon}^{u_{0}, \eta}(A):=\left\{u \in \mathcal{P} C_{\varepsilon}(\Omega): u(\varepsilon i)=u_{0}(\varepsilon i) \text { for any } i \in \mathbb{Z}^{d} \cap\left(\varepsilon^{-1} A^{\eta}\right)\right\},
\end{gathered}
$$


and

$$
m_{\varepsilon}^{\eta}\left(u_{0}, A\right):=\inf \left\{E_{\varepsilon}(u, A): u \in \mathcal{P} C_{\varepsilon}^{u_{0}, \eta}(A)\right\} .
$$

It is easy to show that $m_{\varepsilon}^{\eta}\left(u_{0}\right)$ is increasing in $\eta$ and bounded uniformly in $\eta$ and $\varepsilon$.

TheOREm 4.9. Let $\left(c_{i, \xi}^{\varepsilon}\right)$ satisfy $(\mathrm{H} 1)-(\mathrm{H} 3)$ and let $E_{\varepsilon}$ and $m_{\varepsilon}^{\eta}\left(u_{0}\right)$ be defined by (4.1) and (4.19), respectively. Given a sequence $\varepsilon_{n} \rightarrow 0$ let $\left(\varepsilon_{n_{k}}\right)$ and $E(u, A)$ be as in Theorem 4.2. Then

$$
\lim _{\eta \rightarrow 0} \limsup _{k \rightarrow+\infty} m_{\varepsilon_{n_{k}}}^{\eta}\left(u_{0}, A\right)=m\left(u_{0}, A\right)
$$

where

$$
\begin{array}{r}
m\left(u_{0}, A\right):=\inf \left\{E(u, A): u \in B V(A ;\{ \pm 1\}), u(x)=u_{0}(x)\right. \\
\text { in a neighborhood of } \partial A\} .
\end{array}
$$

Proof. Let $\eta>0$ be fixed and let $u_{k} \in \mathcal{P} C_{\varepsilon_{n_{k}}}^{u_{0}, \eta}(A)$ such that $E_{\varepsilon_{n_{k}}}\left(u_{k}, A\right) \leq$ $m_{\varepsilon_{n_{k}}}^{\eta}\left(u_{0}, A\right)+k^{-1}$. Since, by the coerciveness assumption (H1), $\mathcal{H}^{d-1}\left(S_{u_{k}} \cap A\right) \leq C$, then, by Theorem 2.1, up to extracting a subsequence, $u_{k}$ converge strongly in $L^{1}(A)$ to some $u \in B V(A ;\{ \pm 1\})$. Moreover it is easy to show that $u=u_{0}$ on $A^{\eta}$. Hence, by Theorem 4.2 , we get

$$
m\left(u_{0}, A\right) \leq E(u, A) \leq \liminf _{k} E_{\varepsilon_{n_{k}}}\left(u_{k}, A\right) \leq \liminf _{k} m_{\varepsilon_{n_{k}}}^{\eta}\left(u_{0}, A\right) .
$$

By the arbitrariness of $\eta$ it follows that

$$
m\left(u_{0}, A\right) \leq \lim _{\eta \rightarrow 0} \limsup _{k \rightarrow+\infty} m_{\varepsilon_{n_{k}}}^{\eta}\left(u_{0}, A\right)
$$

It remains to prove the opposite inequality. To this end, we fix $\delta>0$ and $u \in$ $B V(A ;\{ \pm 1\})$ such that $u=u_{0}$ in a neighborhood of $\partial A$ and $E(u, A) \leq m\left(u_{0}, A\right)+\delta$. Let $u_{k} \rightarrow u$ strongly in $L^{1}(A)$ such that $\lim _{k} E_{\varepsilon_{n_{k}}}\left(u_{k}, A\right)=E(u, A)$ and let $\eta>0$ be such that $u=u_{0}$ in $A^{2 \eta}$. We may then argue as in the proof of Proposition 4.5 in order to modify the values of $u_{k}$ in $A^{\eta}$ without increasing the energy too much. More precisely, for any $\delta^{\prime}>0$ we can select open sets $A_{k, \delta^{\prime}} \in \mathcal{A}^{\text {reg }}(\Omega)$ such that $A \backslash A^{2 \eta} \subset \subset A_{k, \delta^{\prime}} \subset \subset A \backslash A^{\eta}$ and the functions $v_{k, \delta^{\prime}}:=\chi_{A_{k, \delta^{\prime}}} u_{k}+\left(1-\chi_{A_{k, \delta^{\prime}}}\right) u_{0}$ satisfy

(i) $v_{k, \delta^{\prime}} \in \mathcal{P} C_{\varepsilon}^{u_{0}, \eta}(A)$;

(ii) $v_{k, \delta^{\prime}} \rightarrow u$ strongly in $L^{1}(A)$;

(iii) $E_{\varepsilon_{n_{k}}}\left(v_{k, \delta^{\prime}}, A\right) \leq E_{\varepsilon_{n_{k}}}\left(u_{k}, A\right)+E_{\varepsilon_{n_{k}}}\left(u_{0}, A^{2 \eta}\right)+C \delta^{\prime}+O(1)+C_{\delta^{\prime}}\left\|u_{k}-u_{0}\right\|_{L^{1}\left(A^{2 \eta}\right)}$.

Hence, since $\limsup _{k \rightarrow+\infty} E_{\varepsilon_{n_{k}}}\left(u_{0}, A^{2 \eta}\right) \leq C \mathcal{H}^{d-1}\left(S_{u_{0}} \cap A^{2 \eta}\right)$, we get

$$
\limsup _{k \rightarrow+\infty} E_{\varepsilon_{n_{k}}}\left(v_{k, \delta^{\prime}}, A\right) \leq E(u, A)+C \mathcal{H}^{d-1}\left(S_{u_{0}} \cap A^{2 \eta}\right)+C \delta^{\prime} .
$$

By the arbitrariness of $\delta^{\prime}$ and since $\limsup _{\eta \rightarrow 0} \mathcal{H}^{d-1}\left(S_{u_{0}} \cap A^{2 \eta}\right)=0$, we infer that

$$
\lim _{\eta \rightarrow 0} \limsup _{k \rightarrow+\infty} m_{\varepsilon_{n_{k}}}^{\eta}\left(u_{0}, A\right) \leq E(u, A) \leq m\left(u_{0}, A\right)+\delta .
$$

The conclusion follows by the arbitrariness of $\delta$. 
Remark 4.10. It can be shown that if the density function $\varphi(x, \nu)$ in Theorem 4.2 is continuous in $\bar{A} \times S^{d-1}$, then

$$
m\left(u_{0}, A\right)=\inf \left\{\int_{S_{u_{A, u_{0}}} \cap \bar{A}} \varphi\left(x, \nu_{u_{A, u_{0}}}\right) d \mathcal{H}^{d-1}: u \in B V(A ;\{ \pm 1\})\right\},
$$

where

$$
u_{A, u_{0}}(x):= \begin{cases}u(x) & \text { if } x \in A, \\ u_{0}(x) & \text { if } x \notin A .\end{cases}
$$

Moreover in this case it can be proved that

$$
m\left(u_{0}, A\right)=\lim _{k \rightarrow+\infty} \min \left\{E_{\varepsilon_{n_{k}}}^{u_{0}}(u, A): u \in \mathcal{P} C_{\varepsilon_{n_{k}}}(A)\right\},
$$

where

$$
E_{\varepsilon}^{u_{0}}(u, A):=\sum_{\xi \in \mathbb{Z}^{d}} \sum_{\varepsilon i \in A_{\varepsilon}} c_{i, \xi}^{\varepsilon} \varepsilon^{d-1}\left|u_{A, u_{0}}(\varepsilon(i+\xi))-u_{A, u_{0}}(\varepsilon i)\right|
$$

(see Theorems 4.2 and 4.4 in [7]).

In fact Example 4.8 shows that $\varphi(\cdot, \nu)$ is not necessarily continuous.

We conclude the section presenting the convergence result of minimum problems with prescribed volume fraction. More precisely, we consider minimum problems of the type

$$
m_{\varepsilon}:=\min \left\{E_{\varepsilon}(u): \#\left\{i \in \Omega_{\varepsilon}: u_{i}=1\right\}=l_{\varepsilon}\right\},
$$

where $l_{\varepsilon} \in\left\{0, \ldots, \# \Omega_{\varepsilon}\right\}$. We suppose that there exists $p \in[0,1]$ such that

$$
\lim _{\varepsilon \rightarrow 0} l_{\varepsilon}\left(\# \Omega_{\varepsilon}\right)^{-1}=p
$$

(this is not restrictive, up to passing to a subsequence). Set

$$
\mathcal{P} C_{\varepsilon}^{l_{\varepsilon}}(\Omega):=\left\{u \in \mathcal{P} C_{\varepsilon}(\Omega): \#\left\{i \in \Omega_{\varepsilon}: u_{i}=1\right\}=l_{\varepsilon}\right\}
$$

and let $E_{\varepsilon}^{l_{\varepsilon}}: L^{1}(\Omega) \rightarrow[0,+\infty]$ be defined by

$$
E_{\varepsilon}^{l_{\varepsilon}}(u)= \begin{cases}E_{\varepsilon}(u) & \text { if } u \in \mathcal{P} C_{\varepsilon}^{l_{\varepsilon}}(\Omega) \\ +\infty & \text { otherwise in } L^{1}(\Omega)\end{cases}
$$

with $E_{\varepsilon}$ as in (4.1). The following $\Gamma$-convergence theorem holds.

TheOrem 4.11. Let $\left(c_{i, \xi}^{\varepsilon}\right)$ satisfy $(\mathrm{H} 1)-(\mathrm{H} 3)$ and let $l_{\varepsilon}$ satisfy (4.20). Given any sequence $\varepsilon_{n} \rightarrow 0$, let $\left(\varepsilon_{n_{k}}\right)$ and $E(u)$ be as in Theorem 4.2. Then the functionals $E_{\varepsilon_{n_{k}}}^{l_{\varepsilon_{n_{k}}}}$ $\Gamma$-converge with respect to the $L^{1}(\Omega)$ strong topology to the functional $E^{p}: L^{1}(\Omega) \rightarrow$ $[0,+\infty]$ defined as

$$
E^{p}(u)= \begin{cases}E(u) & \text { if }|\{u=1\}|=p|\Omega| \\ +\infty & \text { otherwise in } L^{1}(\Omega) .\end{cases}
$$

Copyright $\odot$ by SIAM. Unauthorized reproduction of this article is prohibited. 
Proof. For the sake of notation, in what follows we drop the dependence on the sequence $n_{k}$. Let $u_{\varepsilon} \in \mathcal{P} C_{\varepsilon}^{l_{\varepsilon}}(\Omega)$ converge to $u$ strongly in $L^{1}(\Omega)$ such that $\liminf _{\varepsilon} E_{\varepsilon}\left(u_{\varepsilon}\right)<+\infty$. By (4.20) and taking into account that $\varepsilon^{d} \# \Omega_{\varepsilon} \rightarrow|\Omega|$, we immediately infer that $|\{u=1\}|=p|\Omega|$. In addition, Theorem 4.2 yelds that

$$
\liminf E_{\varepsilon}^{l_{\varepsilon}}\left(u_{\varepsilon}\right) \geq E^{p}(u)
$$

It remains to prove the $\Gamma$-limsup inequality. Thanks to the density result stated in Theorem A.1 and Remark A.2, it is enough to consider $u \in B V(\Omega ;\{ \pm 1\})$ with $|\{u=1\}|=p|\Omega|$ and $S_{u}$ a polyhedral set. Let $u_{\varepsilon}$ be a recovery sequence for $E(u)$. Note that, arguing as in the proof of Proposition 4.5 and taking into account that $E_{\varepsilon}(u, A)$ is negligible as $\varepsilon \rightarrow 0$ for any open set $A \subset \Omega \backslash S_{u}$, we could replace $u_{\varepsilon}$ by $\chi_{A_{\varepsilon}} u_{\varepsilon}+\left(1-\chi_{A_{\varepsilon}}\right) u$, where $A_{\varepsilon}$ is a suitable neighborhood of $S_{u}$, still obtaining a recovery sequence for $E(u)$. Hence, it is not restrictive to assume $u_{\varepsilon}=u$ in $\Omega \backslash \bar{S}_{\delta}$, where $S_{\delta}$ is a $\delta$-neighborhood of $S_{u}$ for some suitable $\delta>0$ such that $\left|\Omega \backslash \bar{S}_{\delta}\right|>0$.

Having set $\tilde{l}_{\varepsilon}=\#\left\{i \in \Omega_{\varepsilon}: u_{\varepsilon}(\varepsilon i)=1\right\}$, we have that $\lim _{\varepsilon \rightarrow 0}\left|\tilde{l}_{\varepsilon}-l_{\varepsilon}\right| \varepsilon^{d}=0$. If $\tilde{l}_{\varepsilon}=l_{\varepsilon}$ for infinite infinitesimal values of $\varepsilon$, there is nothing left to prove. Assume on the contrary that $\tilde{l}_{\varepsilon}>l_{\varepsilon}$ (the other case being totally symmetric). Let $h_{\varepsilon}:=\left(\tilde{l}_{\varepsilon}-l_{\varepsilon}\right)^{\frac{1}{d}}$, $\bar{h}_{\varepsilon}:=\left[h_{\varepsilon}\right]$, where we denote by $[t]$ the integer part of $t \in \mathbb{R}$. Note that $\varepsilon h_{\varepsilon} \rightarrow 0$ and $0<h_{\varepsilon}^{d}-\bar{h}_{\varepsilon}^{d} \leq d h_{\varepsilon}^{d-1}$. Setting $G_{\varepsilon}^{\delta}:=\{u=1\} \cap\left(\Omega \backslash \bar{S}_{\delta}\right)$, we choose $i_{\varepsilon} \in \Omega_{\varepsilon}$ such that $Q_{\varepsilon}:=\varepsilon i_{\varepsilon}+\left[0, \bar{h}_{\varepsilon} \varepsilon\right)^{d} \subset G_{\varepsilon}^{\delta}$ and let $J_{\varepsilon} \subset\left(G_{\varepsilon}^{\delta} \backslash Q_{\varepsilon}\right) \cap \varepsilon \mathbb{Z}^{d}$ be such that $\# J_{\varepsilon}=h_{\varepsilon}^{d}-\bar{h}_{\varepsilon}^{d}$. We thus set

$$
v_{\varepsilon}(\varepsilon i)= \begin{cases}-1 & \text { if } \varepsilon i \in Q_{\varepsilon} \cup J_{\varepsilon}, \\ u_{\varepsilon}(\varepsilon i) & \text { otherwise. }\end{cases}
$$

Then, by construction, $v_{\varepsilon} \in \mathcal{P} C_{\varepsilon}^{l_{\varepsilon}}(\Omega), v_{\varepsilon} \rightarrow u$ strongly in $L^{1}(\Omega)$, and moreover

$$
E_{\varepsilon}\left(v_{\varepsilon}\right) \leq E_{\varepsilon}\left(u_{\varepsilon}\right)+C \varepsilon^{d-1}\left(\#\left(\partial Q_{\varepsilon} \cap \varepsilon \mathbb{Z}^{d}\right)+\# J_{\varepsilon}\right) \leq E_{\varepsilon}\left(\tilde{v}_{\varepsilon}\right)+C\left(\varepsilon h_{\varepsilon}\right)^{d-1},
$$

from which we get the conclusion for $d \geq 2$. For $d=1$ we may assume that there exist $i_{\varepsilon} \in \Omega_{\varepsilon}$ and $\eta>0$ independent of $\varepsilon$ such that $\varepsilon i_{\varepsilon} \in S_{u_{\varepsilon}}$ and $\left(\varepsilon i_{\varepsilon}-\eta, \varepsilon i_{\varepsilon}\right] \subset\left\{u_{\varepsilon}=1\right\}$ or $\left[\varepsilon i_{\varepsilon}, \varepsilon i_{\varepsilon}+\eta\right) \subset\left\{u_{\varepsilon}=1\right\}$. Hence a recovery sequence $v_{\varepsilon} \in \mathcal{P} C_{\varepsilon}^{l_{\varepsilon}}(\Omega)$ is given by $v_{\varepsilon}:=u_{\varepsilon}-2 \chi_{\left(\left(i_{\varepsilon}-\tilde{l}_{\varepsilon}+l_{\varepsilon}\right) \varepsilon, \varepsilon i_{\varepsilon}\right]}$ in the first case or $v_{\varepsilon}:=u_{\varepsilon}-2 \chi_{\left[\varepsilon i_{\varepsilon},\left(i_{\varepsilon}+\tilde{l}_{\varepsilon}-l_{\varepsilon}\right) \varepsilon\right)}$ in the second case.

As a consequence of Theorem 4.11, the compactness result stated in Theorem 2.1, and the core properties of $\Gamma$-convergence, we eventually get the following result.

TheOREM 4.12. Under the hypotheses of Theorem 4.11, we get

$$
\lim _{k} \min \left\{E_{\varepsilon_{n_{k}}}: \#\left\{i \in \Omega_{\varepsilon_{n_{k}}}: u_{i}=1\right\}=l_{\varepsilon_{n_{k}}}\right\}=\min \{E(u):|\{u=1\}|=p|\Omega|\} .
$$

Moreover if $\left(u_{k}\right)$ is a converging sequence such that $\#\left\{i \in \Omega_{\varepsilon_{n_{k}}}: u_{i}=1\right\}=l_{\varepsilon_{n_{k}}}$ and

$$
\lim _{k} E_{\varepsilon_{n_{k}}}\left(u_{k}\right)=\lim _{k} \min \left\{E_{\varepsilon_{n_{k}}}: \#\left\{i \in \Omega_{\varepsilon_{n_{k}}}: u_{i}=1\right\}=l_{\varepsilon_{n_{k}}}\right\},
$$

then its limit is a minimizer to $\min \{E(u):|\{u=1\}|=p|\Omega|\}$.

4.3. Generalization: Multibody interactions. In this section we extend the previous results to the case of energies accounting for multibody interactions. More precisely, given $M \in \mathbb{N}$, we consider energies of the form

$$
\mathcal{E}_{\varepsilon}(u)= \begin{cases}\sum_{i_{1}, \ldots, i_{M} \in \Omega_{\varepsilon}} \varepsilon^{d-1} \psi_{\varepsilon}\left(i_{1}, \ldots, i_{M}, u_{i_{1}}, \ldots, u_{i_{M}}\right) & \text { if } u \in \mathcal{P} C_{\varepsilon}(\Omega), \\ +\infty & \text { otherwise in } L^{1}(\Omega) .\end{cases}
$$

Copyright $@$ by SIAM. Unauthorized reproduction of this article is prohibited. 
Here $\psi_{\varepsilon}: \mathbb{Z}^{d M} \times\{-1,1\}^{M} \rightarrow[0,+\infty)$ satisfies the following hypothesis:

$(\mathrm{HM} 0) \min _{\underline{u}} \psi_{\varepsilon}(\underline{i}, \underline{u})=\psi_{\varepsilon}(\underline{i}, \pm \underline{1})=0$ for any $\underline{i} \in \mathbb{Z}^{d M}$ and either $\psi_{\varepsilon}(\underline{i}, \cdot) \equiv 0$ or $\psi_{\varepsilon}(\underline{\underline{i}}, \underline{u})>0$ for all $\underline{u} \neq \pm \underline{1}$, where we have used the notation

$$
\underline{i}:=\left(i_{1}, \ldots, i_{M}\right), \quad \underline{u}:=\left(u_{1}, \ldots, u_{M}\right), \quad \underline{1}=(1, \ldots, 1) .
$$

Hypothesis (HM0) implies that $\mathcal{E}_{\varepsilon}$ has a ferromagnetic behavior, in the sense that

$$
\min \mathcal{E}_{\varepsilon}=\mathcal{E}_{\varepsilon}(\underline{u})=0 \quad \text { if and only if } \quad \underline{u} \equiv \pm 1 .
$$

As for pairwise interactions, through the change of variables $i=i_{1}, \xi_{l}=i_{l+1}-i$, $l \in\{1, \ldots, M-1\}$, we find it convenient to rewrite $E_{\varepsilon}(u)$ for $u \in \mathcal{P} C_{\varepsilon}(\Omega)$ as

$$
\mathcal{E}_{\varepsilon}(u)=\sum_{\left.\underline{\xi} \in \mathbb{Z}^{d(M-1)}\right)} \sum_{i, i+\xi_{1}, \ldots, i+\xi_{M-1} \in \Omega_{\varepsilon}} \varepsilon^{d-1} \psi_{\varepsilon}\left(i, i+\underline{\xi}, u_{i}, u_{i+\underline{\xi}}\right),
$$

where we have used the notation

$$
\underline{\xi}:=\left(\xi_{1}, \ldots, \xi_{M-1}\right), \quad i+\underline{\xi}:=\left(i+\xi_{1}, \ldots, i+\xi_{M-1}\right), \quad u_{i+\underline{\xi}}=\left(u_{i+\xi_{1}}, \ldots, u_{i+\xi_{M-1}}\right) .
$$

The analogue of assumptions (H1)-(H3) is given by the following set of hypotheses:

(HM1) (coerciveness) $\min \left\{\psi_{\varepsilon}(i, i+\underline{\xi}, \underline{u}): \exists l \in\{1, \ldots, M-1\}\right.$ with $\xi_{l}=e_{k}, \quad u_{l+1}=$ $\left.-u_{1}\right\} \geq c>0$ for all $\varepsilon>0$ and $k \in\{1, \ldots, d\}$;

(HM2) (growth) setting $c_{\varepsilon, \underline{\xi}}=\sup _{i, \underline{u}} \psi_{\varepsilon}(i, \underline{\xi}, \underline{u})$, it holds $\lim \sup _{\varepsilon \rightarrow 0} \sum_{\underline{\xi \in \mathbb{Z} d(M-1)}}|\underline{\xi}| c_{\varepsilon, \underline{\xi}}$ $<+\infty$

(HM3) (decay) for all $\delta>0$ there exists $R_{\delta}>0$ such that $\lim _{\sup _{\varepsilon \rightarrow 0}} \sum_{|\underline{\xi}|>R_{\delta}}|\underline{\xi}| c_{\varepsilon, \underline{\xi}}<$ $\delta$

where we denote by $|\underline{\xi}|$ the usual euclidean norm of $\underline{\xi}$ as a vector in $\mathbb{R}^{d(M-1)}$.

We define also a local version of the functionals in (4.21), by setting for any $A \in \mathcal{A}(\Omega)$

$$
\mathcal{E}_{\varepsilon}(u, A)= \begin{cases}\sum_{i_{1}, \ldots, i_{M} \in A_{\varepsilon}} \varepsilon^{d-1} \psi_{\varepsilon}\left(i_{1}, \ldots, i_{M}, u_{i_{1}}, \ldots, u_{i_{M}}\right) & \text { if } u \in \mathcal{P} C_{\varepsilon}(\Omega), \\ +\infty & \text { otherwise in } L^{1}(\Omega) .\end{cases}
$$

By applying the abstract method exploited in the previous section, we can show that all the possible $\Gamma$-limits of $\mathcal{E}_{\varepsilon}(u, A)$ satisfy the hypotheses of Theorem 2.2 and prove the following theorem, which is the analogue of Theorem 4.2. We omit its proof since it follows, step by step, the proof of Theorem 4.2 without any significant changes.

TheOrem 4.13. Let $\psi_{\varepsilon}$ satisfy (HM0)-(HM3) and let $\mathcal{E}_{\varepsilon}$ be defined by (4.21). Then for any sequence $\varepsilon_{n} \rightarrow 0$ there exist a subsequence $\left(\varepsilon_{n_{k}}\right)$ and a function $\varphi$ : $\Omega \times S^{d-1} \rightarrow[0,+\infty)$ such that the functionals $\mathcal{E}_{\varepsilon_{n_{k}}} \Gamma$-converge with respect to the $L^{1}(\Omega)$ strong topology to the functional $\mathcal{E}: L^{1}(\Omega) \rightarrow[0,+\infty]$ defined by

$$
\mathcal{E}(u)= \begin{cases}\int_{S_{u}} \varphi\left(x, \nu_{u}\right) d \mathcal{H}^{d-1} & \text { if } u \in B V(\Omega ;\{ \pm 1\}), \\ +\infty & \text { otherwise. }\end{cases}
$$

Moreover for any $u \in B V(\Omega ;\{ \pm 1\})$ and $A \in \mathcal{A}^{\text {reg }}(\Omega)$ there holds

$$
\Gamma-\lim _{k} \mathcal{E}_{\varepsilon_{n_{k}}}(u, A)=\mathcal{E}(u, A):=\int_{S_{u} \cap A} \varphi\left(x, \nu_{u}\right) d \mathcal{H}^{d-1} .
$$

Copyright $@$ by SIAM. Unauthorized reproduction of this article is prohibited. 
Under periodicity assumptions on the interaction potentials, the analogue of the homogenization result stated in Theorem 4.7 holds true. More precisely, assume that (HMP) $\psi_{\varepsilon}(i, i+\underline{\xi}, \underline{u})=\psi(i, \underline{\xi}, \underline{u})$, where, for any $\underline{\xi} \in \mathbb{Z}^{d(M-1)}$ and $\underline{u} \in\{-1,1\}^{M}$, $\psi(\cdot, \underline{\xi}, \underline{u})$ is $[0, l]^{d}$-periodic for some $l \in \mathbb{N}$.

In this case assumptions (HM2) and (HM3) reduce to $\left(\mathrm{HM}^{\prime}\right) \sum_{\underline{\xi} \in \mathbb{Z}^{d}(M-1)}|\xi| \sup _{\underline{u}} \psi(i, \underline{\xi}, \underline{u})<+\infty$ for any $\underline{i} \in\{0, \ldots, l-1\}^{d}$.

THEOREM 4.14. Let $\psi_{\varepsilon}$ satisfy (HM0), (HM1), (HMP), and (HM2') and let $\mathcal{E}_{\varepsilon}$ be defined by $(4.21)$. Then the functionals $\mathcal{E}_{\varepsilon} \Gamma$-converge with respect to the $L^{1}(\Omega)$ strong topology, as $\varepsilon \rightarrow 0$, to the homogenized functional $\mathcal{E}_{\text {hom }}: L^{1}(\Omega) \rightarrow[0,+\infty]$ defined by

$$
\mathcal{E}_{\text {hom }}(u)= \begin{cases}\int_{S_{u}} \varphi_{\text {hom }}\left(\nu_{u}\right) d \mathcal{H}^{d-1} & \text { if } u \in B V(\Omega ;\{ \pm 1\}), \\ +\infty & \text { otherwise, }\end{cases}
$$

where

$$
\begin{array}{r}
\varphi_{\text {hom }}(\nu)=\lim _{T \rightarrow+\infty} T^{1-d} \inf \left\{\sum_{\underline{\xi} \in \mathbb{Z}^{d(M-1)}} \sum_{i \in \mathbb{Z}^{d} \cap T Q_{\nu}} \psi\left(i, \underline{\xi}, u_{i}, u_{i+\underline{\xi}}\right): u \in \mathcal{P} C_{1}\left(\mathbb{R}^{d}\right),\right. \\
\left.u(i)=u_{0, \nu}(i) \text { for } i \notin T Q_{\nu}\right\} .
\end{array}
$$

The proof follows step by step that of Theorem 4.7, taking into account that the convergence of boundary value problems stated in Theorem 4.9 also holds in the present case of multibody interactions.

The following example provides an approximation of the usual $\ell^{1}$-anisotropic perimeter in $\mathbb{R}^{2}$ by means of energies accounting for nearest-neighbor 2-body and 3-body interactions. The related asymptotics highlights how the presence of nonpairwise interaction potentials may induce formation of special optimal patterns among those arising in the simpler central case.

Example 4.15. Let $\overline{\mathcal{E}}_{\varepsilon}(u)$ be defined as in (4.21) with $d=2$ and

$$
\psi_{\varepsilon}\left(i, j, k, u_{i}, u_{j}, u_{k}\right)=\left|u_{i}-u_{j}\right|+\left|u_{i}-u_{k}\right|+\left|u_{i}-u_{j}\right|\left|u_{i}-u_{k}\right|
$$

if $(i, j, k)=\left(i, i+e_{1}, i+e_{2}\right)$ and 0 otherwise. Note that $\overline{\mathcal{E}}_{\varepsilon}$ is obtained by adding the 3-body interaction potential $\left|u_{i}-u_{i+e_{1}}\right|\left|u_{i}-u_{i+e_{2}}\right|$ in the definition (4.6) of the functionals $G_{\varepsilon}$. In fact $\overline{\mathcal{E}}_{\varepsilon}$ and $G_{\varepsilon}$ share the same $\Gamma$-limit, that is $\overline{\mathcal{E}}_{\varepsilon}(u) \Gamma$-converge to the functional $\overline{\mathcal{E}}(u)$ defined as in $(4.22)$, with $\varphi_{h o m}(\nu)=|\nu|_{1}$. In order to prove this result, since the functionals $\overline{\mathcal{E}}_{\varepsilon}$ satisfy the hypotheses of Theorem 4.14, it suffices to check that $\Gamma-\lim _{\varepsilon \rightarrow 0} \overline{\mathcal{E}}_{\varepsilon}\left(u_{0, \nu}\right)=\overline{\mathcal{E}}\left(u_{0, \nu}\right)$ for any $\nu \in S^{d-1}$. The $\Gamma$-liminf inequality follows at once by neglecting the 3 -body interaction potential, that is, using the inequality

$$
\mathcal{E}_{\varepsilon}(u) \geq G_{\varepsilon}(u) .
$$

Conversely, it is easy to check that, given $N_{\varepsilon} \in \mathbb{N}$ such that $\lim _{\varepsilon} \varepsilon N_{\varepsilon}=0$ and set $\delta_{\varepsilon}:=\varepsilon N_{\varepsilon}$, then the functions $u_{\varepsilon} \in \mathcal{P} C_{\delta_{\varepsilon}}(\Omega) \subset \mathcal{P} C_{\varepsilon}(\Omega)$ defined by $u_{\varepsilon}\left(\delta_{\varepsilon} i\right)=u_{0, \nu}\left(\delta_{\varepsilon} i\right)$, $i \in \mathbb{Z}^{d}$, are a recovery sequence for $G_{\varepsilon}$ at $u_{0, \nu}$. A direct computation shows that the 3-body potential gives a positive contribution to $\mathcal{E}_{\varepsilon}\left(u_{\varepsilon}\right)$ only on the "corners" of the interface of $u_{\varepsilon}$, that is when the interface of $u_{\varepsilon}$ changes direction. Hence, whenever $\nu \notin\left\{e_{1}, e_{2}\right\}$ we get

$$
\mathcal{E}_{\varepsilon}\left(u_{\varepsilon}\right)=G\left(u_{\varepsilon}\right)+C N_{\varepsilon}^{-1} .
$$


Thus, $u_{\varepsilon}$ is a recovery sequence for $\mathcal{E}_{\varepsilon}$ at $u_{0, \nu}$ if and only if $\lim _{\varepsilon \rightarrow 0} N_{\varepsilon}=+\infty$. The computation above shows that the 3 -body potential does not give any contribution to the limiting energy but acts as a selector of the optimal states of $G_{\varepsilon}$.

5. Approximation of nonlocal continuum functionals. In this section we show that if hypothesis (H3) is violated, then the $\Gamma$-limits of energies as in (4.1) can be nonlocal functionals. We will focus on the approximation of two relevant examples of nonlocal functionals: fractional perimeters and Ohta-Kawasaki-type energies.

5.1. Fractional perimeters. We first introduce a notion of fractional perimeters, suitably defined in order to deal with nonlocal contributions only inside the target set $\Omega$.

Definition 5.1. Let $E \subset \Omega$ and $0<s<1$. The fractional s-perimeter inside $\Omega$ $P_{s}(E ; \Omega)$ of $E$ is defined as follows:

$$
P_{s}(E, \Omega):=\int_{E} \int_{E^{c} \cap \Omega} \frac{1}{|y-x|^{d+s}} d x d y .
$$

Fractional perimeters and related minimization problems were first introduced in [16] and since then they have been largely investigated (see, for instance, [24] in the framework of phase transitions problems, and [10] for the convergence of scaled $s$ perimeters to the classical one). Our definition of fractional $s$-perimeter differs from the usual one, introduced by [16], as it neglects the contribution of couples in $E \times \Omega^{c}$. Starting with the pioneering work [2] nonlocal type energies as in (5.1) have been studied in connection with both phase transitions and dislocations problems.

Note that $P_{s}(E, \Omega)$ is half of the fractional Sobolev space seminorm $\left|\chi_{E}\right|_{W^{s, 1}(\Omega)}$, where $\chi_{E}$ denotes the characteristic function of $E$. Moreover, if $u:=\chi_{E}-\chi_{E^{c}}$ then

$$
P_{s}(E, \Omega)=\frac{1}{4}|u|_{W^{s, 1}(\Omega)}:=\int_{\Omega} \int_{\Omega} \frac{|u(y)-u(x)|}{|y-x|^{d+s}} d x d y .
$$

We recall that if $E$ is a set of finite perimeter in $\Omega$, then $P_{s}(E, \Omega)$ is also finite, since in general $B V(\Omega) \subset W^{s, 1}(\Omega)$ for any $s \in(0,1)$. We will approximate functionals defined on $B V(\Omega ;\{ \pm 1\})$ and of the form

$$
\int_{S_{u}} \varphi\left(\nu_{u}\right) d \mathcal{H}^{d-1}+\int_{\Omega} \int_{\Omega} \frac{|u(y)-u(x)|}{|y-x|^{d+s}} d x d y .
$$

We may approximate the first term in (5.2) by ferromagnetic energies satisfying the hypotheses of Theorem 4.7. In order to approximate the second term in (5.2), the idea is simply to discretize it. In fact, setting $Q_{i}^{\varepsilon}=\varepsilon i+[0, \varepsilon)^{d}$ for $i \in \mathbb{Z}^{d}$, we have

$$
\begin{aligned}
& \int_{\Omega} \int_{\Omega} \frac{|u(y)-u(x)|}{|y-x|^{d+s}} d x d y=\sum_{i, j} \int_{\left(\Omega \cap Q_{i}^{\varepsilon}\right) \times\left(\Omega \cap Q_{j}^{\varepsilon}\right)} \frac{|u(y)-u(x)|}{|y-x|^{d+s}} d x d y \\
= & \sum_{i \neq j \in \Omega_{\varepsilon}} \varepsilon^{2 d} \frac{|u(\varepsilon j)-u(\varepsilon i)|}{|\varepsilon(j-i)|^{d+s}}+o(1)=\sum_{\xi \in \mathbb{Z}^{d} \backslash\{0\}} \sum_{i, i+\xi \in \Omega_{\varepsilon}} \varepsilon^{d-1} c_{\xi}^{\varepsilon}\left|u_{i+\xi}-u_{i}\right|+o(1),
\end{aligned}
$$

where $c_{\xi}^{\varepsilon}:=\frac{\varepsilon^{1-s}}{|\xi|^{d+s}}$.

Let $\bar{E}_{\varepsilon}: L^{1}(\Omega) \rightarrow[0,+\infty]$ be defined by

$$
\bar{E}_{\varepsilon}(u):=E_{\varepsilon}(u)+\sum_{\xi \in \mathbb{Z}^{d} \backslash\{0\}} \sum_{i, i+\xi \in \Omega_{\varepsilon}} \varepsilon^{d-s} \frac{\left|u_{i+\xi}-u_{i}\right|}{|\xi|^{d+s}},
$$

Copyright (c) by SIAM. Unauthorized reproduction of this article is prohibited. 
where $E_{\varepsilon}$ satisfies the hypotheses of Theorem 4.7 , and set

$$
E_{\varepsilon}^{n l}(u):=\sum_{\xi \in \mathbb{Z}^{d} \backslash\{0\}} \sum_{i, i+\xi \in \Omega_{\varepsilon}} \varepsilon^{d-s} \frac{\left|u_{i+\xi}-u_{i}\right|}{|\xi|^{d+s}} .
$$

In the next proposition we show that $E_{\varepsilon}^{n l}$ is essentially a continuous perturbation of $E_{\varepsilon}$.

Proposition 5.2. Let $u_{\varepsilon}: \Omega_{\varepsilon} \rightarrow\{ \pm 1\}$ be such that $u_{\varepsilon} \rightarrow u$ in $L^{1}(\Omega)$ and $\sup _{\varepsilon} \mathcal{H}^{d-1}\left(S_{u_{\varepsilon}}\right)<+\infty$. Then $u \in B V(\Omega ; \pm 1)$ and

$$
\lim _{\varepsilon \rightarrow 0} E_{\varepsilon}^{n l}\left(u_{\varepsilon}\right)=\int_{\Omega} \int_{\Omega} \frac{|u(y)-u(x)|}{|y-x|^{d+s}} d x d y .
$$

Proof. Let us extend $u_{\varepsilon}$ outside $\Omega_{\varepsilon}$ by setting $u_{\varepsilon}(\varepsilon i)=1$ if $i \in \mathbb{Z}^{d} \backslash \varepsilon^{-1} \Omega$. Since $\partial \Omega$ is Lipschitz, we still have that $\sup _{\varepsilon} \mathcal{H}^{d-1}\left(S_{u_{\varepsilon}}\right)<+\infty$, which implies that for any $h \in \mathbb{R}^{d}$ it holds

$$
\int_{\mathbb{R}^{d}}\left|u_{\varepsilon}(x+h)-u_{\varepsilon}(x)\right| d x \leq C|h|
$$

with $C>0$ independent of $\varepsilon$ and $h$. Given $\delta>0$, we split $E_{\varepsilon}^{n l}\left(u_{\varepsilon}\right)$ into two terms accounting for the interactions at distances greater and less than $\delta$, respectively, i.e.,

$$
\begin{aligned}
E_{\varepsilon}^{n l}\left(u_{\varepsilon}\right) & =\sum_{\xi \in \mathbb{Z}^{d} \cap B_{\delta / \varepsilon} \backslash\{0\}} \sum_{i, i+\xi \in \Omega_{\varepsilon}} \varepsilon^{d-s} \frac{\left|\left(u_{\varepsilon}\right)_{i+\xi}-\left(u_{\varepsilon}\right)_{i}\right|}{|\xi|^{d+s}} \\
& +\sum_{\xi \in \mathbb{Z}^{d} \backslash B_{\delta / \varepsilon}} \sum_{i, i+\xi \in \Omega_{\varepsilon}} \varepsilon^{d-s} \frac{\left|\left(u_{\varepsilon}\right)_{i+\xi}-\left(u_{\varepsilon}\right)_{i}\right|}{|\xi|^{d+s}}=: I_{\varepsilon, \delta}^{1}+I_{\varepsilon, \delta}^{2} .
\end{aligned}
$$

It is easy to show that

$$
I_{\varepsilon, \delta}^{2}=\int_{\Omega \times \Omega \cap\{|y-x|>\delta\}} \frac{\left|u_{\varepsilon}(y)-u_{\varepsilon}(x)\right|}{|y-x|^{d+s}} d x d y+o(1)
$$

from which we get that

$$
\lim _{\varepsilon \rightarrow 0} I_{\varepsilon, \delta}^{2}=\int_{\Omega \times \Omega \cap\{|y-x|>\delta\}} \frac{|u(y)-u(x)|}{|y-x|^{d+s}} d x d y .
$$

We proceed now by estimating $I_{\varepsilon, \delta}^{1}$. It can be easily shown that

$$
I_{\varepsilon, \delta}^{1} \leq \sum_{\xi \in \mathbb{Z}^{d} \cap B_{\delta / \varepsilon} \backslash\{0\}} \frac{\varepsilon^{-s}}{|\xi|^{d+s}} \int_{\mathbb{R}^{d}}\left|u_{\varepsilon}(x+\varepsilon \xi)-u_{\varepsilon}(x)\right| d x .
$$

Hence, by (5.4), we get

$$
I_{\varepsilon, \delta}^{1} \leq C \varepsilon^{1-s} \sum_{\xi \in \mathbb{Z}^{d} \cap B_{\delta / \varepsilon} \backslash\{0\}} \frac{1}{|\xi|^{d+s-1}} .
$$

Copyright (c) by SIAM. Unauthorized reproduction of this article is prohibited. 
Note that

$$
\begin{aligned}
& \sum_{\xi \in \mathbb{Z}^{d} \cap B_{\delta / \varepsilon} \backslash\{0\}} \frac{1}{|\xi|^{d+s-1}}=\sum_{k=1}^{[\delta / \varepsilon]-1} \sum_{\xi \in \mathbb{Z}^{d} \cap\left(B_{k+1} \backslash B_{k}\right)} \frac{1}{|\xi|^{d+s-1}} \\
& \leq \sum_{k=1}^{[\delta / \varepsilon]-1} \frac{\#\left(\mathbb{Z}^{d} \cap\left(B_{k+1} \backslash B_{k}\right)\right)}{k^{d+s-1}} \leq C \sum_{k=1}^{[\delta / \varepsilon]-1} \frac{1}{k^{s}} \leq C\left(\frac{\delta}{\varepsilon}\right)^{1-s} \text {. }
\end{aligned}
$$

Thus, from (5.7) we deduce that

$$
I_{\varepsilon, \delta}^{1} \leq C \delta^{1-s}
$$

Eventually, by (5.5), (5.6), and (5.8), we infer that

$$
\lim _{\varepsilon \rightarrow 0} E_{\varepsilon}^{n l}\left(u_{\varepsilon}\right)=\int_{\Omega \times \Omega \cap\{|y-x|>\delta\}} \frac{|u(y)-u(x)|}{|y-x|^{d+s}} d x d y+O\left(\delta^{1-s}\right)
$$

and the conclusion follows by the arbitrariness of $\delta>0$.

From Proposition 5.2 we derive the following $\Gamma$-convergence result.

TheOREM 5.3. Let $E_{\varepsilon}$ satisfy the hypotheses of Theorem 4.7. Then the functionals $\bar{E}_{\varepsilon}$, defined in (5.3), $\Gamma$-converge with respect to the $L^{1}(\Omega)$ strong topology to the functional $\bar{E}: L^{1}(\Omega) \rightarrow[0,+\infty]$ defined by

$$
\bar{E}(u)= \begin{cases}\int_{S_{u}} \varphi_{h o m}\left(\nu_{u}\right) d \mathcal{H}^{d-1}+\int_{\Omega} \int_{\Omega} \frac{|u(y)-u(x)|}{|y-x|^{d+s}} d x d y & \text { if } u \in B V(\Omega ;\{ \pm 1\}), \\ +\infty & \text { otherwise, }\end{cases}
$$

where $\varphi_{\text {hom }}$ is defined by (4.16).

Proof. The thesis follows by Theorem 4.7 and Proposition 5.2.

Remark 5.4. Note that the functional $\bar{E}_{\varepsilon}$ defined in (5.3) is of the form (4.1) with $c_{i, \xi}^{\varepsilon}=c_{\xi}(i)+\frac{\varepsilon^{1-s}}{|\xi|^{d+s}}$ if $\xi \in\left(\mathbb{Z}^{d} \cap B_{R / \varepsilon}\right) \backslash\{0\}$ and $c_{i, \xi}^{\varepsilon}=c_{\xi}(i)$ otherwise, where $R:=\operatorname{diam}(\Omega)$ and $c_{\xi}(\cdot)$ satisfy hypotheses $\left(\mathrm{H}^{\prime}\right)$ and (H2'). A direct computation shows that for any $R>0$

$$
\limsup _{\varepsilon \rightarrow 0} \sum_{\xi \in \mathbb{Z}^{d} \cap B_{R / \varepsilon} \backslash\{0\}} \frac{\varepsilon^{1-s}}{|\xi|^{d+s}}<+\infty ; \quad \limsup _{\varepsilon \rightarrow 0} \sum_{\xi \in \mathbb{Z}^{d} \cap\left(B_{R / \varepsilon} \backslash B_{R / 2 \varepsilon}\right)} \frac{\varepsilon^{1-s}}{|\xi|^{d+s}} \geq C>0 .
$$

Hence hypotheses (H1), (H2) are satisfied, while (H3) is violated.

Remark 5.5 (generalization). The approximation result of Theorem 5.3 can be extended to a general class of nonlocal functionals, without any significant changes in its proof. More precisely, let $K: \Omega \times \Omega \rightarrow[0,+\infty)$ be such that $K \in C^{1}(\Omega \times \Omega \backslash$ $\{x=y\})$ and $K(x, y) \leq C|y-x|^{-d-s}$ in a neighborhood of $\{x=y\}$ for some $s \in(0,1)$. Moreover let $H_{\varepsilon}: L^{1}(\Omega) \rightarrow[0,+\infty]$ be defined by

$$
H_{\varepsilon}(u):=E_{\varepsilon}(u)+\sum_{\xi \in \mathbb{Z}^{d} \backslash\{0\}} \sum_{i, i+\xi \in \Omega_{\varepsilon}} \varepsilon^{2 d} K(\varepsilon i, \varepsilon(i+\xi))\left|u_{i+\xi}-u_{i}\right|,
$$

Copyright (c) by SIAM. Unauthorized reproduction of this article is prohibited. 
where $E_{\varepsilon}$ satisfies the hypotheses of Theorem 4.7 , and let $H: L^{1}(\Omega) \rightarrow[0,+\infty]$ be defined by

$H(u)= \begin{cases}\int_{S_{u}} \varphi_{h o m}\left(\nu_{u}\right) d \mathcal{H}^{d-1}+\int_{\Omega} \int_{\Omega} K(x, y)|u(y)-u(x)| d x d y & \text { if } u \in B V(\Omega ;\{ \pm 1\}), \\ +\infty & \text { otherwise, }\end{cases}$

where $\varphi_{\text {hom }}$ is defined by (4.16). Then Theorem 5.3 still holds with $H_{\varepsilon}$ in place of $\bar{E}_{\varepsilon}$ and $H$ in place of $\bar{E}$.

5.2. Ohta-Kawasaki-type energies. A canonical mathematical model in the studies of energy-driven pattern forming systems is based on the following energy first proposed by Ohta-Kawasaki (see [23]):

$$
\mathcal{E}_{\varepsilon}(u)=\varepsilon \int_{\Omega}|\nabla u|^{2} d x+\frac{1}{\varepsilon} \int_{\Omega}\left(1-u^{2}\right)^{2} d x+\gamma_{0} \int_{\Omega} \int_{\Omega} G(x, y) u(x) u(y) d x d y .
$$

Here $u$ is an $H^{1}(\Omega)$ phase parameter describing the density distribution of the components ( $u=-1$ stands for one phase, $u=+1$ for the other), subject to the constraint $m=f_{\Omega} u d x$, that is the difference of the phases' volume fractions is prescribed, and $G$ is the Green's function of the Laplacian with Neumann boundary conditions on $\partial \Omega$. The first two integrals in (5.9) form the so called Modica-Mortola energy which $\Gamma$ converges as $\varepsilon \rightarrow 0$ to the perimeter functional (see [19]). It can be easily shown that the nonlocal term in (5.9) is an $L^{1}$ continuous perturbation of the Modica-Mortola energy. Hence, the functionals $\mathcal{E}_{\varepsilon} \Gamma$-converge with respect to the $L^{1}(\Omega)$ norm to the functional $\mathcal{E}: L^{1}(\Omega) \rightarrow[0,+\infty]$ given by

$$
\mathcal{E}(u):= \begin{cases}\frac{8}{3} \mathcal{H}^{d-1}\left(S_{u}\right)+\gamma_{0} \int_{\Omega} \int_{\Omega} G(x, y) u(x) u(y) d x d y & \text { if } u \in B V(\Omega ;\{ \pm 1\}), \\ +\infty & \text { otherwise. }\end{cases}
$$

We provide a variational approximation of an anisotropic version of the functional in (5.10), namely, the functional finite on $B V(\Omega ;\{ \pm 1\})$ and defined by

$$
\int_{S_{u}}\left|\nu_{u}\right|_{1} d \mathcal{H}^{d-1}\left(S_{u}\right)+\gamma_{0} \int_{\Omega} \int_{\Omega} G(x, y) u(x) u(y) d x d y .
$$

We may "discretize" the nonlocal term as in the previous section, that is

$$
\begin{gathered}
\int_{\Omega} \int_{\Omega} G(x, y) u(x) u(y) d x d y=\sum_{i, j} \int_{\left(\Omega \cap Q_{i}^{\varepsilon}\right) \times\left(\Omega \cap Q_{j}^{\varepsilon}\right)} G(x, y) u(x) u(y) d x d y \\
=\sum_{i \neq j \in \Omega_{\varepsilon}} \varepsilon^{2 d} G(\varepsilon i, \varepsilon j) u(\varepsilon i) u(\varepsilon j)+o(1) \\
=\sum_{\xi \in \mathbb{Z}^{d} \backslash\{0\}} \sum_{i, i+\xi \in \Omega_{\varepsilon}} \varepsilon^{2 d} G(\varepsilon i, \varepsilon(i+|\xi|)) u_{i+\xi} u_{i}+o(1) .
\end{gathered}
$$

We note that the Green's function $G$ satisfies

$$
|G(x, y)| \leq \frac{C}{|x-y|^{d-2}} \leq \frac{C}{|x-y|^{d^{\prime}}}
$$

Copyright $\odot$ by SIAM. Unauthorized reproduction of this article is prohibited. 
for constants $C$ depending on $\Omega$ and for some $d-2 \leq d^{\prime}<d$. In particular

$$
\int_{\Omega} \int_{\Omega}|G(x, y)| d x d y<+\infty .
$$

A discrete version of (5.11) is provided by the following estimate

$$
\begin{aligned}
\sum_{\xi \in \mathbb{Z}^{d} \backslash\{0\}} \sum_{i, i+\xi \in \Omega_{\varepsilon}} \varepsilon^{2 d} G(\varepsilon i, \varepsilon(i+|\xi|)) & \leq C \sum_{\xi \in \mathbb{Z}^{d} \cap B_{r / \varepsilon} \backslash\{0\}} \varepsilon^{d} \frac{1}{|\varepsilon \xi|^{d^{\prime}}} \\
& =C \varepsilon^{d-d^{\prime}} \sum_{\xi \in \mathbb{Z}^{d} \cap B_{r / \varepsilon} \backslash\{0\}} \frac{1}{|\xi|^{d^{\prime}}} \leq C,
\end{aligned}
$$

where we have set $r:=\operatorname{diam}(\Omega)$.

Let $\hat{F}_{\varepsilon}: L^{1}(\Omega) \rightarrow[0,+\infty]$ be defined by

$$
\hat{F}_{\varepsilon}(u):=\left\{\begin{array}{lr}
-\sum_{|j-i|=1} \varepsilon^{d} u_{i} u_{j}+\gamma_{0} \sum_{\xi \in \mathbb{Z}^{d} \backslash\{0\}} \sum_{i, i+\xi \in \Omega_{\varepsilon}} \varepsilon^{2 d+1} G(\varepsilon i, \varepsilon(i+|\xi|)) u_{i+\xi} u_{i} \\
=: \hat{F}_{\varepsilon}^{l o c}(u)+\hat{F}_{\varepsilon}^{n l}(u) & \text { if } u \in \mathcal{P} C_{\varepsilon}(\Omega), \\
+\infty & \text { otherwise. }
\end{array}\right.
$$

Note that $\hat{F}_{\varepsilon}$ is of the form (3.1). Moreover, we point out that, since $G(\cdot, \cdot)$ changes sign, $\hat{F}_{\varepsilon}$ mixes ferromagnetic and antiferromagnetic interactions. Hence the uniform states $u \equiv \pm 1$ are not absolute minimizers of $\hat{F}_{\varepsilon}$. Nevertheless, estimate (5.12) yields that $\hat{F}_{\varepsilon}^{n l}(u)$ vanishes uniformly with respect to $u$ as $\varepsilon$ goes to 0 , and then

$$
\hat{F}_{\varepsilon}( \pm 1)=\min \hat{F}_{\varepsilon}+O(\varepsilon)=\hat{F}_{\varepsilon}^{l o c}( \pm 1)+O(\varepsilon) .
$$

We then consider the scaled energies

$$
\hat{E}_{\varepsilon}(u):=\frac{\hat{F}_{\varepsilon}(u)-\hat{F}_{\varepsilon}^{l o c}( \pm 1)}{\varepsilon},
$$

which can be written as

$$
\hat{E}_{\varepsilon}(u)=\left\{\begin{array}{lc}
\sum_{|j-i|=1} \varepsilon^{d-1}\left|u_{j}-u_{i}\right|+\gamma_{0} \sum_{\xi \in \mathbb{Z}^{d} \backslash\{0\}} \sum_{i, i+\xi \in \Omega_{\varepsilon}} \varepsilon^{2 d} G(\varepsilon i, \varepsilon(i+|\xi|)) u_{i+\xi} u_{i} \\
=: \hat{E}_{\varepsilon}^{l o c}(u)+\hat{E}_{\varepsilon}^{n l}(u) & \text { if } u \in \mathcal{P} C_{\varepsilon}(\Omega), \\
+\infty & \text { otherwise. }
\end{array}\right.
$$

Arguing as in the proof of Proposition 5.2, the following result can be proved.

Proposition 5.6. Let $u_{\varepsilon}: \Omega_{\varepsilon} \rightarrow\{ \pm 1\}$ be such that $u_{\varepsilon} \rightarrow u$ in $L^{1}(\Omega)$. Then

$$
\lim _{\varepsilon \rightarrow 0} \hat{E}_{\varepsilon}^{n l}\left(u_{\varepsilon}\right)=\int_{\Omega} \int_{\Omega} G(x, y) u(x) u(y) d x d y .
$$

By Proposition 5.6, we derive the following $\Gamma$-convergence result. Its proof is straightforward.

Copyright (c) by SIAM. Unauthorized reproduction of this article is prohibited. 
THEOREM 5.7. The functionals $\hat{E}_{\varepsilon}$, defined in (5.13), $\Gamma$-converge with respect to the $L^{1}(\Omega)$ strong topology to the functional $\hat{E}: L^{1}(\Omega) \rightarrow[0,+\infty]$ defined by

$$
\hat{E}(u):= \begin{cases}\int_{S_{u}}\left|\nu_{u}\right|_{1} d \mathcal{H}^{d-1}\left(S_{u}\right)+\gamma_{0} \int_{\Omega} \int_{\Omega} G(x, y) u(x) u(y) d x d y & \\ +\infty & \text { if } u \in B V(\Omega ;\{ \pm 1\}), \\ + \text { otherwise. }\end{cases}
$$

Proof. Notice that $\hat{E}_{\varepsilon}^{l o c}(u)=G_{\varepsilon}(u)$ for $u \in \mathcal{P} C_{\varepsilon}(\Omega)$, where $G_{\varepsilon}$ is defined in (4.6), and we have already recalled that in $[3$, Theorem 4$]$ it was proved that

$$
\Gamma-\lim _{\varepsilon \rightarrow 0} G_{\varepsilon}(u)= \begin{cases}\int_{S_{u}}\left|\nu_{u}\right|_{1} \mathcal{H}^{d-1} & \text { if } u \in B V(\Omega ;\{ \pm 1\}), \\ +\infty & \text { otherwise. }\end{cases}
$$

Hence, the thesis follows by Proposition 5.6.

The result in Theorem 5.7 can be extended to the periodic case, when $\Omega=\mathbb{T}^{d}$, and to functions satisfying a mean constraint. More precisely, assume $\varepsilon=\frac{1}{n}$ and, given $l_{n} \in\left\{0, \ldots n^{d}\right\}$, set

$$
\begin{aligned}
\mathcal{P} C_{n}^{l_{n}}\left(\mathbb{T}^{d}\right):=\left\{u: \frac{1}{n} \mathbb{Z}^{d} \rightarrow\{-1,1\}, u[0,1)^{d}\right. \text {-periodic and } & \\
& \left.\#\left\{i \in([0, n) \cap \mathbb{Z})^{d}: u_{i}=1\right\}=l_{n}\right\} .
\end{aligned}
$$

We assume that there exists $p \in[0,1]$ such that

$$
\lim _{n \rightarrow+\infty} n^{-d} l_{n}=p
$$

Then, consider the functionals $E_{n}^{l_{n}}: L^{1}\left(\mathbb{T}^{d}\right) \rightarrow[0,+\infty]$ defined as

$$
E_{n}^{l_{n}}(u)=\left\{\begin{array}{lc}
\sum_{|j-i|=1} n^{1-d}\left|u_{j}-u_{i}\right|+\gamma_{0} \sum_{\xi \in \mathbb{Z}^{d} \backslash\{0\}} \sum_{i \in n Q \cap \mathbb{Z}^{d}} n^{-2 d} G\left(\frac{i}{n}, \frac{(i+|\xi|)}{n}\right) u_{i+\xi} u_{i} \\
+\infty & \text { if } u \in \mathcal{P} C_{n}^{l_{n}}\left(\mathbb{T}^{d}\right), \\
+\infty \text { otherwise }
\end{array}\right.
$$

where $G$ is the Green's functions for the Laplacian with periodic boundary conditions. Then, by following the same steps of the proof of Theorem 4.11 and taking into account Theorem A.1 and Remark A.2, the following result can be proved.

TheOREM 5.8. Let $\left(l_{n}\right)$ satisfy (5.14). Then, the functionals $E_{n}^{l_{n}}$, defined in (5.15), $\Gamma$-converge with respect to the $L^{1}\left(\mathbb{T}^{d}\right)$ strong topology to the functional $E^{p}$ : $L^{1}\left(\mathbb{T}^{d}\right) \rightarrow[0,+\infty]$ defined by

$$
E^{p}(u):=\left\{\begin{array}{rr}
\int_{S_{u}}\left|\nu_{u}\right|_{1} d \mathcal{H}^{d-1}\left(S_{u}\right)+\gamma_{0} \int_{\mathbb{T}^{d}} \int_{\mathbb{T}^{d}} G(x, y) u(x) u(y) d x d y & \\
& \text { if } u \in B V\left(\mathbb{T}^{d} ;\{ \pm 1\}\right) \text { and }|\{u=1\}|=p, \\
+\infty & \text { otherwise. }
\end{array}\right.
$$

Copyright (c) by SIAM. Unauthorized reproduction of this article is prohibited. 
The previous result allows us to deduce the existence of "lamellar-type" local minimizers for the discrete energies $E_{n}^{p_{n}}$ : according to a definition introduced in [1], we say that a function $u \in \mathcal{P} C_{n}^{l_{n}}\left(\mathbb{T}^{d}\right)$ is an isolated $L^{1}$-local minimizer for the functional $E_{n}^{l_{n}}$ if there exists $\delta>0$ such that

$$
E_{n}^{l_{n}}(u) \leq E_{n}^{l_{n}}(v), \forall v \in \mathcal{P} C_{n}^{l_{n}}\left(\mathbb{T}^{d}\right) \text { with } 0<\min _{\tau \in \frac{1}{n} \mathbb{Z}^{d}}\|u-v(\cdot+\tau)\|_{L^{1}\left(\mathbb{T}^{d}\right)} \leq \delta .
$$

An analogous definition of isolated local minimizers can be given in the continuum for the functional $E^{p}(u)$.

In $[1$, Proposition 5.6] the following result was proved: given $p \in(0,1)$, set

$$
L_{k}:=T^{d-1} \times \cup_{l=1}^{k}\left[\frac{l-1}{k}, \frac{l-1}{k}+\frac{p}{k}\right], \quad k \in \mathbb{N}
$$

and denote by $\mathcal{L}_{p, k}$ the collection of all sets which may be obtained from $L_{k}$ by translations and relabeling of coordinates. Then, for any $\gamma_{0}>0$ and $L \in \mathcal{L}_{p, k}$, the function $u_{L}:=\chi_{L}-\chi_{L^{c}}$ is an isolated $L^{1}$-local minimizer for the functional $\mathcal{E}^{p}: L^{1}\left(\mathbb{T}^{d}\right) \rightarrow[0,+\infty]$ defined by

$$
\mathcal{E}^{p}(u):= \begin{cases}\mathcal{H}^{d-1}\left(S_{u}\right)+\gamma_{0} \int_{\mathbb{T}^{d}} \int_{\mathbb{T}^{d}} G(x, y) u(x) u(y) d x d y & \\ & \text { if } u \in B V\left(\mathbb{T}^{d} ;\{ \pm 1\}\right) \text { and }|\{u=1\}|=p, \\ +\infty & \text { otherwise }\end{cases}
$$

for $k$ large enough. Since $\mathcal{H}^{d-1}\left(S_{u}\right) \leq \int_{S_{u}}\left|\nu_{u}\right|_{1} d \mathcal{H}^{d-1}$ and the equality holds for $u_{L}$, we get that $u_{L}$ is also an isolated $L^{1}$-local minimizer for the functional $E^{p}$. Hence, as a straightforward consequence of Theorems 2.3 and 5.8, we deduce the following result.

Theorem 5.9 (local minimality of lamellae). There exists $k_{0} \in \mathbb{N}$ such that for any $k \in \mathbb{N}, k>k_{0}$, and $L \in \mathcal{L}_{p, k}$ there exist $n_{0} \in \mathbb{N}$ and a family $\left\{u_{n}\right\}_{n>n_{0}}$ of isolated local minimizers of $E_{n}^{l_{n}}$ such that $u_{n} \rightarrow u_{L}$ strongly in $L^{1}\left(\mathbb{T}^{d}\right)$, as $n \rightarrow+\infty$, where $u_{L}:=\chi_{L}-\chi_{L^{c}}$.

6. General criterion for a ferromagnetic behavior. In this section we consider energies of the type (3.1) in the homogeneous case $c_{i, \xi}^{\varepsilon}=c_{\xi}$ for all $i$ and $\xi$, without constraints on the sign of such constants. Our main goal is to provide a general criterion which ensures that such energies still have a ferromagnetic behavior, that is the ground states are still the uniform states $u \equiv 1$ and $u \equiv-1$ and the continuum limit of the scaled energies is an interfacial energy of the form (4.4). We restrict our analysis to the case in which only short-range interactions are taken into account, that is there exists $R>0$ such that $c_{\xi}=0$ if $|\xi|>R$. Hence, we consider energies of the form

$$
F_{\varepsilon}(u)=-\sum_{\xi \in \mathbb{Z}^{d} \cap B(0, R)} \sum_{i, i+\xi \in \Omega_{\varepsilon}} c_{\xi} \varepsilon^{d} u_{i} u_{i+\xi}
$$

on $\mathcal{P} C_{\varepsilon}(\Omega)$ and equal to $+\infty$ otherwise in $L^{1}(\Omega)$. In order to introduce and better explain the ferromagnetic criterion we are going to define, we first consider two onedimensional examples. 
Example 6.1 (nearest neighbor and next-to-nearest neighbor interactions). Let $\Omega$ $=(0,1)$ and let $F_{\varepsilon}$ be the energy accounting for nearest and next-to-nearest neighbor interactions defined by

$$
F_{\varepsilon}(u):=-c_{1} \sum_{i,(i+1) \in \mathbb{Z} \cap\left(0, \varepsilon^{-1}\right)} \varepsilon u_{i} u_{i+1}-c_{2} \sum_{i,(i+2) \in \mathbb{Z} \cap\left(0, \varepsilon^{-1}\right)} \varepsilon u_{i} u_{i+2} .
$$

Note that, given $\lambda \in \mathbb{R}$, we may rewrite $F_{\varepsilon}$ as

$$
F_{\varepsilon}(u)=\sum_{i \in \mathbb{Z} \cap\left(0, \varepsilon^{-1}-2\right)} \varepsilon\left(-c_{1}\left(\lambda u_{i} u_{i+1}+(1-\lambda) u_{i+1} u_{i+2}\right)-c_{2} u_{i} u_{i+2}\right)+O(\varepsilon),
$$

where the additional infinitesimal term is due to the energetic contribution of the interactions near 0 and 1 . Set, for $v:\{0,1,2\} \rightarrow\{-1,1\}$,

$$
F_{\text {cell }}^{2}(v):=-c_{1}\left(\lambda v_{0} v_{1}+(1-\lambda) v_{1} v_{2}\right)-c_{2} v_{0} v_{2} .
$$

Then, a condition which guarantees that the uniform states -1 and 1 are the only two minimizers of $F_{\varepsilon}$ up to lower order terms is the following

$$
F_{\text {cell }}^{2}(v)>F_{\text {cell }}^{2}( \pm 1)=-c_{1}-c_{2} \quad \forall v:\{0,1,2\} \rightarrow\{-1,1\}, v \not \equiv \pm 1 .
$$

Indeed, if (6.3) is satisfied, formula (6.2) immediately infers that

$$
F_{\varepsilon}( \pm 1)=\min F_{\varepsilon}+O(\varepsilon) .
$$

It is easy to show that (6.3) is satisfied with $\lambda=\frac{1}{2}$ if and only if $c_{1}, c_{2} \in A:=$ $\left\{c_{1}>0,2 c_{2}>-c_{1}\right\}$. Moreover, easy computations show that

(1) if $c_{1}, c_{2} \in B:=\left\{c_{1}<0,2 c_{2}>c_{1}\right\}$, we have two ground states given by the alternating states $(-1)^{i}$ and $(-1)^{i-1}$

(2) if $c_{1}, c_{2} \in C:=\left\{c_{1}<0,2 c_{2}<c_{1}\right\} \cup\left\{c_{1}>0,2 c_{2}<-c_{1}\right\}$, we have four ground states given by $(-1)^{\left[\frac{i+k}{2}\right]}, k=0,1,2,3$;

(3) if $c_{1}, c_{2} \in \bar{A} \cap \bar{B}=\left\{c_{1}=0, c_{2}>0\right\}$, we have four ground states given by the uniform states 1 and -1 and the alternating states given by $(-1)^{i}$ and $(-1)^{i-1}$; if $c_{1}, c_{2} \in \bar{A} \cap \bar{C}=\left\{c_{1}>0,2 c_{2}=-c_{1}\right\}$, we have six ground states given by the uniform states 1 and -1 and the states $(-1)^{\left[\frac{i+k}{2}\right]}, k=0,1,2,3$; if $c_{1}, c_{2} \in \bar{B} \cap \bar{C}=\left\{c_{1}<0,2 c_{2}=c_{1}\right\}$, we have six ground states given by the alternating states $(-1)^{i}$ and $(-1)^{i-1}$ and the states $(-1)^{\left[\frac{i+k}{2}\right]}, k=0,1,2,3$.

Example 6.2. (nearest neighbor, next-to-nearest neighbor, and next-to-next-tonearest neighbor interactions) Let $\Omega=(0,1)$ and let $F_{\varepsilon}$ be the energy accounting for nearest, next-to-nearest, and next-to-next-to-nearest neighbor interactions, defined by

$$
\begin{aligned}
F_{\varepsilon}(u):= & -c_{1} \sum_{i,(i+1) \in \mathbb{Z} \cap\left(0, \varepsilon^{-1}\right)} \varepsilon u_{i} u_{i+1}-c_{2} \sum_{i,(i+2) \in \mathbb{Z} \cap\left(0, \varepsilon^{-1}\right)} \varepsilon u_{i} u_{i+2} \\
& -c_{3} \sum_{i,(i+3) \in \mathbb{Z} \cap\left(0, \varepsilon^{-1}\right)} \varepsilon u_{i} u_{i+3} .
\end{aligned}
$$

Following the same strategy as the previous example, given $\lambda, \alpha, \beta \in \mathbb{R}$, we may rewrite $F_{\varepsilon}$ as 


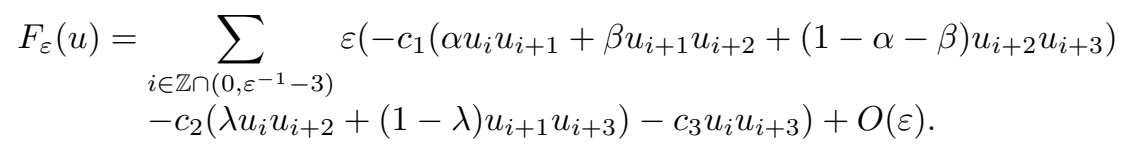

Set, for $v:\{0,1,2,3\} \rightarrow\{-1,1\}$

$F_{\text {cell }}^{3}(v):=-c_{1}\left(\alpha v_{0} v_{1}+\beta v_{1} v_{2}+(1-\alpha-\beta) v_{2} v_{3}\right)-c_{2}\left(\lambda v_{0} v_{2}+(1-\lambda) v_{1} v_{3}\right)-c_{3} v_{0} v_{3}$.

In this case the uniform states -1 and 1 are the only two minimizers of $F_{\varepsilon}$ up to lower order term if there holds

$$
F_{\text {cell }}^{3}(v)>F_{\text {cell }}^{3}( \pm 1)=-c_{1}-c_{2}-c_{3} \quad \forall v:\{0,1,2,3\} \rightarrow\{-1,1\}, v \not \equiv \pm 1 .
$$

If $c_{1}, c_{2}, c_{3} \in\left\{c_{1}>-3 c_{3}>0,3 c_{2}>-c_{1}-3 c_{3}\right\} \cup\left\{c_{1}=0, c_{2}>0, c_{3}>0\right\}$ one can check that (6.4) is satisfied. Note that for other values of $c_{1}, c_{2}$, and $c_{3}$ it is in principle possible that neither the uniform states 1 and -1 nor different periodic states are minimizers of $F_{\text {cell }}^{3}$. In this case one could further push the argument before as follows: given $M \in \mathbb{N}, M \geq 3$, the three sets of constants $\left\{\alpha_{1}^{k}, \ldots, \alpha_{M-k+1}^{k}\right\}$, $k=1,2,3$, satisfying $\sum_{j=1}^{M-k+1} \alpha_{j}^{k}=1$ for any $k$, one can rewrite $F_{\varepsilon}$ as

$$
F_{\varepsilon}(u)=\sum_{i \in \mathbb{Z} \cap\left(0, \varepsilon^{-1}-M\right)} \varepsilon\left(-\sum_{k=1}^{3} c_{l} \sum_{j=1}^{M-k+1} \alpha_{j}^{k} u_{i+j-1} u_{i+j+k-1}\right)+O(\varepsilon)
$$

and define, for $v:\{0,1, \ldots, M\} \rightarrow\{-1,1\}$,

$$
F_{c e l l}^{M}(v):=-\sum_{k=1}^{3} c_{k} \sum_{j=1}^{M-k+1} \alpha_{j}^{k} v_{j-1} v_{j+k-1}
$$

Then, as before, the uniform states -1 and 1 are the only ground states if there holds

$$
F_{\text {cell }}^{M}(v)>F_{\text {cell }}^{M}( \pm 1)=-c_{1}-c_{2}-c_{3} \quad \forall v:\{0,1, \ldots, M\} \rightarrow\{-1,1\}, v \not \equiv \pm 1 .
$$

We now generalize the argument exploited in the previous examples. Let $F_{\varepsilon}$ be defined by (6.1). Note that, for any $M \in \mathbb{N}, M \geq R$, we may rewrite $F_{\varepsilon}$ as

$$
F_{\varepsilon}(u)=\sum_{\varepsilon i \in \Omega_{\varepsilon}^{M}} \varepsilon^{d}\left(-\sum_{|\xi| \leq R} c_{\xi} \sum_{j, j+\xi \in C_{M}} \phi_{M}(j, \xi) u^{i+j} u^{i+j+\xi}\right)+O(\varepsilon),
$$

where $C_{M}:=[0, M]^{d} \cap \mathbb{Z}^{d}, \Omega_{\varepsilon}^{M}:=\left\{i \in \mathbb{Z}^{d}: \varepsilon i+\varepsilon C_{M} \subset \Omega\right\}$, and $\phi_{M}: C_{M} \times \mathbb{Z}^{d} \rightarrow \mathbb{R}$ satisfies for any fixed $\xi \in \mathbb{Z}^{d}$

$$
\sum_{j, j+\xi \in C_{M}} \phi_{M}(j, \xi)=1
$$

Note that the additional infinitesimal term in (6.5) is due to the energetic contribution of the interactions near the boundary of $\Omega$. Set, for $v: C_{M} \rightarrow\{ \pm 1\}$,

$$
F_{c e l l}^{M}(v):=-\sum_{|\xi| \leq R} c_{\xi} \sum_{j, j+\xi \in C_{M}} \phi_{M}(j, \xi) v^{j} v^{j+\xi} .
$$

Copyright $@$ by SIAM. Unauthorized reproduction of this article is prohibited. 
Definition 6.3 (ferromagnetic criterion). We say the family $\left\{c_{\xi}: \quad \xi \in \mathbb{Z}^{d} \cap\right.$ $B(0, R)\}$ has a ferromagnetic behavior if there exists $M \in \mathbb{N}, M \geq R$, and $\phi_{M}$ : $C_{M} \times \mathbb{Z}^{d} \rightarrow \mathbb{R}$ such that

$$
\sum_{j, j+\xi \in C_{M}} \phi_{M}(j, \xi)=1 \quad \forall \xi \in \mathbb{Z}^{d}
$$

and

$$
F_{\text {cell }}^{M}(v)>F_{\text {cell }}^{M}( \pm 1)=-\sum_{|\xi| \leq R} c_{\xi} \quad \forall v: C_{M} \rightarrow\{ \pm 1\}, v \not \equiv \pm 1 .
$$

Formula (6.6) is a sufficient condition for the minimality of the uniform states 1 and -1 . Indeed, if (6.6) is satisfied, formula (6.5) immediately infers that

$$
F_{\varepsilon}( \pm 1)=\min F_{\varepsilon}+O(\varepsilon) .
$$

We may, then, consider the scaled energies

$$
E_{\varepsilon}(u)=\frac{F_{\varepsilon}(u)-F_{\varepsilon}( \pm 1)}{\varepsilon},
$$

which can be written on $\mathcal{P} C_{\varepsilon}(\Omega)$ as

$$
E_{\varepsilon}(u)=\sum_{\varepsilon i \in \Omega_{\varepsilon}^{M}} \varepsilon^{d-1}\left(\sum_{|\xi| \leq R} c_{\xi} \sum_{j, j+\xi \in C_{M}}\left(1-\phi_{M}(j, \xi)\right) u^{i+j} u^{i+j+\xi}\right)+O(1) .
$$

Let us set

$$
E_{\text {cell }}^{M}(v):=F_{\text {cell }}^{M}(v)-F_{\text {cell }}^{M}( \pm 1)=\sum_{|\xi| \leq R} c_{\xi} \sum_{j, j+\xi \in C_{M}}\left(1-\phi_{M}(j, \xi)\right) v^{j} v^{j+\xi} .
$$

Moreover, with a little abuse of notation, set for $u \in \mathcal{P} C_{\varepsilon}(\Omega)$ and $i \in \Omega_{\varepsilon}$

$$
E_{\text {cell }}^{M}\left(u, i+C_{M}\right):=E_{\text {cell }}^{M}(u(1 / \varepsilon \cdot+i)) .
$$

Note that we get

$$
E_{\varepsilon}(u)=\sum_{\varepsilon i \in \Omega_{\varepsilon}^{M}} \varepsilon^{d-1} E_{c e l l}^{M}\left(u, i+C_{M}\right)+O(1) .
$$

In the next proposition we show that the functionals $E_{\varepsilon}$ are equicoercive with respect to the $L^{1}(\Omega)$-topology and their possible $\Gamma$-limits are finite only on $B V(\Omega ;\{ \pm 1\})$.

Proposition 6.4. Assume that the family $\left\{c_{\xi}: \xi \in \mathbb{Z}^{d} \cap B(0, R)\right\}$ has a ferromagnetic behavior, according to Definition 6.3, and let $E_{\varepsilon}$ be defined by (6.7). Let $u_{\varepsilon}$ be such that $\sup _{\varepsilon} E_{\varepsilon}\left(u_{\varepsilon}\right)<+\infty$. Then there exist a subsequence $\varepsilon_{k}$ and $u \in B V(\Omega ;\{ \pm 1\})$ such that $u_{\varepsilon_{k}}$ converge to $u$ strongly in $L^{1}(\Omega)$.

Proof. Let $\left\{\Omega^{k}\right\}$ be an increasing sequence of open sets compactly contained in $\Omega$ such that $\cup_{k \in \mathbb{N}} \Omega^{k}=\Omega$. By (6.6) and (6.9), we have that for any $k \in \mathbb{N}$

$$
\begin{aligned}
\mathcal{H}^{d-1}\left(S\left(u_{\varepsilon}\right) \cap \Omega^{k}\right) & \leq C \varepsilon^{d-1} \#\left\{i \in \mathbb{Z}^{d} \cap \frac{1}{\varepsilon} \Omega^{k}: E_{\text {cell }}^{M}\left(u_{\varepsilon}, i+C_{M}\right)>0\right\} \\
& \leq C E_{\varepsilon}\left(u_{\varepsilon}\right) \leq C
\end{aligned}
$$

By Theorem 2.1 and by a diagonalization argument we get the conclusion.

Copyright (c) by SIAM. Unauthorized reproduction of this article is prohibited. 
Now we pass to show that the $\Gamma$-limit of $E_{\varepsilon}$ is an interfacial energy of the form (4.4). Since it is not our purpose in this paper to investigate boundary layer effects, we assume that $\Omega=\mathbb{T}^{d}, \varepsilon=\frac{1}{n}, n \in \mathbb{N}$. Moreover from now on we will use the notation $E_{n}$ in place of $E_{\frac{1}{n}}$. In this periodic setting $E_{n}$ turns out to be defined on all $[0,1)^{d}$-periodic functions $u: \frac{1}{n} \mathbb{Z}^{d} \rightarrow\{-1,1\}$ as

$$
\begin{aligned}
E_{n}(u) & =\sum_{\xi \in \mathbb{Z}^{d} \cap B(0, R)} \sum_{i \in \mathbb{Z}^{d} \cap n Q} c_{\xi} n^{1-d}\left(1-u_{i} u_{i+\xi}\right) \\
& =\sum_{i \in \mathbb{Z}^{d} \cap n Q} n^{1-d} E_{\text {cell }}^{M}\left(u, i+C_{M}\right)
\end{aligned}
$$

is equal to $+\infty$ otherwise in $L^{1}\left(\mathbb{T}^{d}\right)$, and

$$
E_{n}( \pm 1)=\min E_{n}=0 .
$$

Theorem 6.5. Assume that the family $\left\{c_{\xi}: \quad \xi \in \mathbb{Z}^{d} \cap B(0, R)\right\}$ has a ferromagnetic behavior, according to Definition 6.3, and let $E_{n}$ be defined by (6.10). Let $M>R$ and $\phi_{M}: C_{M} \times \mathbb{Z}^{d} \rightarrow \mathbb{R}$ such that (6.6) is satisfied. Then the functionals $E_{n}$ $\Gamma$-converge with respect to the $L^{1}\left(\mathbb{T}^{d}\right)$-topology to the functional $E: L^{1}\left(\mathbb{T}^{d}\right) \rightarrow[0,+\infty]$ defined by

$$
E(u)= \begin{cases}\int_{S(u)} \varphi\left(\nu_{u}\right) d \mathcal{H}^{d-1} & \text { if } u \in B V\left(\mathbb{T}^{d} ;\{ \pm 1\}\right), \\ +\infty & \text { otherwise }\end{cases}
$$

where $\varphi: S^{d-1} \rightarrow[0,+\infty)$ is the restriction to $S^{d-1}$ of a convex and positively homogeneous function of degree one and it is defined by

$$
\varphi(\nu):=\lim _{T \rightarrow+\infty} \frac{1}{T^{d-1}} \min \left\{\sum_{i \in \mathbb{Z}^{d} \cap T Q_{\nu}} E_{\text {cell }}^{M}\left(u, i+C_{M}\right): u \in D\left(T Q_{\nu}\right)\right\},
$$

where $E_{\text {cell }}^{M}$ is defined by (6.8) and

$$
D\left(T Q_{\nu}\right):=\left\{u: \mathbb{Z}^{d} \rightarrow\{-1,1\}: u(i)=u_{0, \nu}(i) \forall i \in \mathbb{Z}^{d} \backslash(T-R) Q_{\nu}\right\} .
$$

Remark 6.6 (bounds). The function $\varphi$ defined in (6.11) satisfies the following bounds

$$
\alpha|\nu|_{1, \infty} \leq \varphi(\nu) \leq \beta|\nu|_{1, \infty} \quad \forall \nu \in S^{d-1},
$$

where $\alpha:=\min \left\{E_{\text {cell }}^{M}(v): v \not \equiv \pm 1\right\}, \beta:=\max \left\{E_{\text {cell }}^{M}(v): v \not \equiv \pm 1\right\}$, and $|\nu|_{1, \infty}:=$ $\max \left\{\sum_{k \neq l}\left|\nu_{k}\right|: l \in\{1, \ldots N\}\right\}$. The proof of the lower bound can be obtained by using a slicing argument along the directions $e_{\bar{l}}$, where $\bar{l} \in \operatorname{argmax}|\nu|_{1, \infty}$, and taking into account that, by (6.6), the transition between the two states 1 and -1 costs a positive energy. The proof of the upper bound can be easily obtained by testing the minimum problem in (6.11) with $u=u_{0, \nu}$ and using again a slicing argument.

The next proposition shows that the definition of $\varphi$ in (6.11) is well-posed. We omit its proof, since it relies on a classical procedure in homogenization theory and use the argument exploited in the construction of the recovery sequence in the proof of Theorem 6.5. 
Proposition 6.7. Let $M>R$ and $\varphi: C_{M} \times \mathbb{Z}^{d} \rightarrow \mathbb{R}$ such that (6.6) is satisfied and let $D\left(T Q_{\nu}\right)$ be defined by (6.12) for all $\nu \in S^{d-1}$ and $T>0$. Then the limit

$$
\lim _{T \rightarrow+\infty} \frac{1}{T^{d-1}} \min \left\{\sum_{i \in \mathbb{Z}^{d} \cap T Q_{\nu}} E_{c e l l}^{M}\left(u, i+C_{M}\right): u \in D\left(T Q_{\nu}\right)\right\}
$$

exists for all $\nu \in S^{d-1}$.

Proof of Theorem 6.5. We first prove the liminf inequality. Let $u_{n} \rightarrow u$ in $L^{1}\left(\mathbb{T}^{d}\right)$ such that $\liminf _{n} E_{n}\left(u_{n}\right)<+\infty$. Up to passing to a subsequence we may assume that $\liminf _{n} E_{n}\left(u_{n}\right)=\lim _{n} E_{n}\left(u_{n}\right)$. Since $u_{n}$ is $[0,1)^{d}$-periodic, then $u$ is $[0,1)^{d}$-periodic. By Proposition 6.4, we immediately deduce that $u \in B V\left(\mathbb{T}^{d} ;\{ \pm 1\}\right)$. We now use a blow-up argument. Let $Q=[0,1)^{d}$ and set

$$
\mu_{n}:=\sum_{i \in \mathbb{Z}^{d} \cap n Q} n^{1-d} E_{c e l l}^{M}\left(u_{n}, i+C_{M}\right) \delta_{\frac{1}{n} i} .
$$

Since $\sup _{n} \mu_{n}(Q)=\sup _{n} E_{n}\left(u_{n}\right)<+\infty$, we may suppose, up to passing to a further subsequence, that there exists a positive finite measure $\mu$ such that $\mu_{n} \rightarrow \mu$. By the Radon-Nikodym theorem, we may decompose $\mu$ into two mutually singular positive measures as

$$
\mu=\psi \mathcal{H}^{d-1}\left\lfloor_{S(u)}+\mu_{s}\right.
$$

The conclusion follows by showing that

$$
\psi\left(x_{0}\right) \geq \varphi\left(\nu_{u\left(x_{0}\right)}\right) \quad \text { for } \mathcal{H}^{d-1}\left\lfloor_{S(u)} \text { a.e. } x_{0} \in S(u) .\right.
$$

For $\mathcal{H}^{d-1}\left\lfloor_{S(u)}\right.$ a.e. $x_{0} \in S(u)$ it holds

(i) $\lim _{\rho \rightarrow 0} \frac{1}{\rho^{d}} \int_{x_{0}+\rho Q_{\nu_{u\left(x_{0}\right)}}^{ \pm}}|u(x)- \pm 1| d x=0$,

(ii) $\lim _{\rho \rightarrow 0} \frac{1}{\rho^{d-1}} \mathcal{H}^{d-1}\left(S(u) \cap\left\{x_{0}+\rho Q_{\nu_{u\left(x_{0}\right)}}\right\}\right)=1$,

(iii) $\lim _{\rho \rightarrow 0} \frac{\mu\left(x_{0}+\rho Q_{\nu_{u\left(x_{0}\right)}}\right)}{\mathcal{H}^{d-1}\left(S(u) \cap\left\{x_{0}+\rho Q_{\nu_{u\left(x_{0}\right)}}\right\}\right)}=\psi\left(x_{0}\right)$.

Fix such an $x_{0} \in S(u)$ and let $\left(\rho_{m}\right)$ be a sequence of positive numbers converging to zero such that $\mu\left(x_{0}+\rho_{m} Q_{\nu_{u\left(x_{0}\right)}}\right)=0$. By (ii) and (iii) we get

$$
\begin{aligned}
\psi\left(x_{0}\right) & =\lim _{m} \frac{\mu\left(x_{0}+\rho_{m} Q_{\nu_{u\left(x_{0}\right)}}\right)}{\rho_{m}^{d-1}} \\
& =\lim _{m} \frac{1}{\rho_{m}^{d-1}} \lim _{n} \sum_{i \in \mathbb{Z}^{d} \cap n\left(x_{0}+\rho_{m} Q_{\nu_{u\left(x_{0}\right)}}\right)} n^{1-d} E_{\text {cell }}^{M}\left(u_{n}, i+C_{M}\right) .
\end{aligned}
$$

Observe that, for any $m$ and $n$ we can find $\rho_{m, n}$ with $\lim _{n} \rho_{m, n}=\rho_{m}$ and $x_{0}^{n} \in \mathbb{Z}^{d}$ with $\frac{1}{n} x_{0}^{n} \rightarrow x_{0}$, such that

$$
\mathbb{Z}^{d} \cap\left(x_{0}^{n}+n \rho_{m, n} Q_{\nu_{u\left(x_{0}\right)}}\right)=\mathbb{Z}^{d} \cap n\left(x_{0}+\rho_{m} Q_{\nu_{u\left(x_{0}\right)}}\right) .
$$

Then

$$
\begin{aligned}
\psi\left(x_{0}\right) & =\lim _{m} \lim _{n} \sum_{i \in \mathbb{Z}^{d} \cap\left(x_{0}^{n}+n \rho_{m, n} Q_{\left.\nu_{u\left(x_{0}\right)}\right)}\right)}\left(n \rho_{m, n}\right)^{1-d} E_{\text {cell }}^{M}\left(u_{n}, i+C_{M}\right) \\
& =\lim _{m} \lim _{n} \sum_{j \in \mathbb{Z}^{d} \cap\left(n \rho_{m, n} Q_{\left.\nu_{u\left(x_{0}\right)}\right)}\right)}\left(n \rho_{m, n}\right)^{1-d} E_{\text {cell }}^{M}\left(u_{n}, x_{0}^{n}+j+C_{M}\right)
\end{aligned}
$$

Copyright (c) by SIAM. Unauthorized reproduction of this article is prohibited. 
Let $v_{m, n}: \frac{1}{n \rho_{m, n}} \mathbb{Z}^{d} \rightarrow\{-1,1\}$ be defined by

$$
v_{m, n}\left(\frac{1}{n \rho_{m, n}} j\right)=u_{n}\left(\frac{1}{n}\left(x_{0}^{n}+j\right)\right), \quad j \in \mathbb{Z}^{d} .
$$

By (i) and since $u_{n} \rightarrow u$ in $L^{1}(Q)$ we get

$$
\lim _{m} \lim _{n} \int_{Q_{\nu_{u\left(x_{0}\right)}}}\left|v_{m, n}-u_{0, \nu_{u\left(x_{0}\right)}}\right| d x=0 .
$$

Hence, by a diagonalization argument, we can find a sequence of positive numbers $\lambda_{k} \rightarrow 0$ and a sequence $v_{k}: \lambda_{k} \mathbb{Z}^{d} \rightarrow\{-1,1\}$ such that $v_{k} \rightarrow u_{0, \nu_{u\left(x_{0}\right)}}$ in $L^{1}\left(Q_{\nu_{u\left(x_{0}\right)}}\right)$ and

$$
\psi\left(x_{0}\right) \geq \lim _{k} \sum_{j \in \mathbb{Z}^{d} \cap \lambda_{k} Q_{\nu_{u\left(x_{0}\right)}}} \lambda_{k}^{d-1} E_{\text {cell }}^{M}\left(v_{k}, j+C_{M}\right) .
$$

By Lemma 6.8, there exists $w_{k}: \lambda_{k} \mathbb{Z}^{d} \rightarrow\{-1,1\}$ such that $w_{k}\left(\lambda_{k} j\right)=u_{\nu}\left(\lambda_{k} j\right)$ if $j \in \mathbb{Z}^{d} \backslash\left(\frac{1}{\lambda_{k}}-R\right) Q_{\nu_{u\left(x_{0}\right)}}$ and

$$
\psi\left(x_{0}\right) \geq \liminf _{k} \sum_{j \in \mathbb{Z}^{d} \cap \frac{1}{\lambda_{k}} Q_{\nu_{u\left(x_{0}\right)}}} \lambda_{k}^{d-1} E_{c e l l}^{M}\left(w_{k}, j+C_{M}\right) .
$$

Eventually the functions $\hat{w}_{k}: \mathbb{Z}^{d} \rightarrow\{-1,1\}$ defined as

$$
\hat{w}_{k}(j):=w_{k}\left(\lambda_{k} j\right), \quad j \in \mathbb{Z}^{d},
$$

belong to $D\left(\frac{1}{\lambda_{k}} Q_{\nu_{u\left(x_{0}\right)}}\right)$ and, setting $T_{k}:=\frac{1}{\lambda_{k}}$, by (4.13), (6.11), and Proposition 6.7 , we get

$$
\psi\left(x_{0}\right) \geq \liminf _{k} T_{k}^{1-d} \sum_{j \in \mathbb{Z}^{d} \cap T_{k} Q_{\nu_{u}\left(x_{0}\right)}} E_{\text {cell }}^{M}\left(\hat{w}_{k}, j+C_{M}\right) \geq \varphi\left(\nu_{u\left(x_{0}\right)}\right) .
$$

We now pass to the construction of a recovery sequence. We perform this construction just for functions $u \in B V\left(\mathbb{T}^{d} ;\{ \pm 1\}\right)$ such that $S(u)$ is a polyhedral set, since the set of these functions is dense in $B V\left(\mathbb{T}^{d} ;\{ \pm 1\}\right)$. We further restrict to the case in which $S(u)$ is the restriction to $Q$ of a hyperplane, since this construction is easily generalized to each face of a polyhedral boundary. Fix, then, $\nu \in S^{d-1}$ and let $u$ be such that $S(u)=\Pi_{\nu} \cap Q$, where $\Pi_{\nu}$ is a hyperplane orthogonal to $\nu$. Without loss of generality we may assume $\Pi_{\nu}=\left\{x \in \mathbb{R}^{d}:\langle x, \nu\rangle=0\right\}$, which corresponds to $u=u_{0, \nu}$ on $Q$. Given $\delta>0$, by Proposition 6.7, there exist $T_{\delta}>0$ and $u_{\delta} \in D\left(T_{\delta} Q_{\nu}\right)$ such that $T_{\delta}>R \delta^{-1}$ and

$$
\frac{1}{T_{\delta}^{d-1}} \sum_{i \in \mathbb{Z}^{d} \cap T Q_{\nu}} E_{c e l l}^{M}\left(u_{\delta}, i+C_{M}\right) \leq \varphi(\nu)+\delta .
$$

Let $\left\{b_{1}, \ldots, b_{d}\right\}$ be an orthonormal base of $\mathbb{R}^{d}$ such that we have $b_{d}=\nu$ and $Q_{\nu}=$ $\left\{x \in \mathbb{R}^{d}:\left|\left\langle x, b_{l}\right\rangle\right|<1 / 2, l=1, \ldots, d\right\}$. For any $j \in \bigoplus_{l=1}^{d-1} \mathbb{Z}\left(T_{\delta}+2 R\right) b_{l}$ set $x_{j}=[j]=$ $\left(\left[j_{1}\right], \ldots,\left[j_{d}\right]\right)$. Then let $u_{n}: \frac{1}{n} \mathbb{Z}^{d} \cap Q \rightarrow\{-1,1\}$ be such that

$$
u_{n}\left(\frac{i}{n}\right)=u_{\delta}\left(i-x_{j}\right) \quad \text { for } i \in \mathbb{Z}^{d} \cap\left(T_{\delta}+2 R\right) Q_{\nu}+x_{j}, j \in \bigoplus_{l=1}^{d-1} \mathbb{Z}\left(T_{\delta}+2 R\right) b_{l},
$$

Copyright $\odot$ by SIAM. Unauthorized reproduction of this article is prohibited. 
and $u \equiv u_{\nu}$ otherwise. It can be easily verified that $u_{n} \rightarrow u$ in $L^{1}(Q)$. Note that, by (6.6), the interactions giving nonzero energetic contribution are only those which are inside the domains of the form $\frac{1}{n}\left(\left(T_{\delta}+2 R\right) Q_{\nu}+x_{j}\right)$ and in an $\frac{R}{n}$-neighborhood of $\partial\left(\frac{1}{n}\left(\left(T_{\delta}+2 R\right) Q_{\nu}+x_{j}\right)\right) \cap \Pi_{\nu} \cap Q$. Summing up all such contributions, we get

$$
\begin{aligned}
E^{\prime \prime}(u) \leq & \limsup _{n} E_{n}\left(u_{n}\right) \leq \mathcal{H}^{d-1}\left(S(u)\left(T_{\delta}+2 R\right)^{1-d} \sum_{i \in \mathbb{Z}^{d} \cap T Q_{\nu}} E_{c e l l}^{M}\left(u_{\delta}, i+C_{M}\right)\right. \\
& +C R \frac{\left(T_{\delta}+2 R\right)^{d-2}}{\left(T_{\delta}+2 R\right)^{d-1}} \leq \mathcal{H}^{d-1}\left(S(u)(1+\delta)^{d-1}(\varphi(\nu)+\delta)+C R \delta .\right.
\end{aligned}
$$

The conclusion follows by letting $\delta$ tend to 0 .

Lemma 6.8. Let $\lambda_{k} \rightarrow 0$ and let $v_{k}: \lambda_{k} \mathbb{Z}^{d} \rightarrow\{-1,1\}$ be such that $v_{k} \rightarrow u_{0, \nu}$ in $L^{1}\left(Q_{\nu}\right)$. Then there exist $w_{k}: \lambda_{k} \mathbb{Z}^{d} \rightarrow\{-1,1\}$ such that $w_{k}\left(\lambda_{k} j\right)=u_{0, \nu}\left(\lambda_{k} j\right)$ if $j \in \mathbb{Z}^{d} \backslash\left(\frac{1}{\lambda_{k}}-R\right) Q_{\nu}$ and

$$
\begin{aligned}
& \liminf _{k} \sum_{j \in \mathbb{Z}^{d} \cap \frac{1}{\lambda_{k}} Q_{\nu}} \lambda_{k}^{d-1} E_{\text {cell }}^{M}\left(w_{k}, j+C_{M}\right) \\
& \leq \liminf _{k} \sum_{j \in \mathbb{Z}^{d} \cap \frac{1}{\lambda_{k}} Q_{\nu}} \lambda_{k}^{d-1} E_{\text {cell }}^{M}\left(v_{k}, j+C_{M}\right) .
\end{aligned}
$$

Proof. Note that $\#\left\{j \in \mathbb{Z}^{d} \cap\left(Q_{\nu} \backslash\left(\frac{1}{\lambda_{k}}-R\right) Q_{\nu}\right): v_{k}\left(\lambda_{k} j\right) \neq u_{0, \nu}\left(\lambda_{k} j\right)\right\} \leq C \lambda_{k}^{2-N}$ if $\left\|v_{k}-u_{0, \nu}\right\|_{L^{1}\left(Q_{\nu}\right)} \leq C \lambda_{k}^{2}$. In this case it can be easily verified that the thesis holds for

$$
w_{k}:=\chi_{\left(1-R \lambda_{k}\right) Q_{\nu}} v_{k}+\left(1-\chi_{\left(1-R \lambda_{k}\right) Q_{\nu}}\right) u_{0, \nu}
$$

Otherwise, we may assume that $\liminf _{k} \frac{1}{\lambda_{k}^{2}}\left\|v_{k}-u_{0, \nu}\right\|_{L^{1}\left(Q_{\nu}\right)}=+\infty$. In this case, set $N_{k}:=\left[\frac{\sqrt{\left\|v_{k}-u_{0, \nu}\right\|_{L^{1}\left(Q_{\nu}\right)}}}{\lambda_{k}}\right]$ and, for $l \in\left\{1, \ldots, N_{k}\right\}$, set
\[ Q_{\nu, l}^{k}:=\left\{x \in Q_{\nu}: \operatorname{dist}\left(x, Q_{\nu}^{c}\right) \geq R l \lambda_{k}\right\} . \]

Then for any $k \in\left\{1, \ldots, N_{k}\right\}$ consider the family of functions $w_{k}^{l}$ defined by

$$
w_{k}^{l}:=\chi_{Q_{\nu, l}^{k}} v_{k}+\left(1-\chi_{Q_{\nu, l}^{k}}\right) u_{0, \nu} .
$$

Arguing as in the proof of Proposition 4.5, we can find $l_{k} \in\left\{1, \ldots, N_{k}\right\}$ such that

$$
\begin{aligned}
& \sum_{j \in \mathbb{Z}^{d} \cap \frac{1}{\lambda_{k}} Q_{\nu}} \lambda_{k}^{d-1} E_{\text {cell }}^{M}\left(w_{k}^{l_{k}}, j+C_{M}\right) \leq \sum_{j \in \mathbb{Z}^{d} \cap \frac{1}{\lambda_{k}} Q_{\nu}} \lambda_{k}^{d-1} E_{\text {cell }}^{M}\left(u_{k}, j+C_{M}\right) \\
&+\sum_{j \in \mathbb{Z}^{d} \cap \frac{1}{\lambda_{k}}\left(Q_{\nu}-\lambda_{k} N_{k} Q_{\nu}\right)} \lambda_{k}^{d-1} E_{\text {cell }}^{M}\left(u_{0, \nu}, j+C_{M}\right)+o(1) .
\end{aligned}
$$

The conclusion follows by choosing $w_{k}:=w_{k}^{l_{k}}$ and noticing that

$$
\lim _{k} \sum_{j \in \mathbb{Z}^{d} \cap \frac{1}{\lambda_{k}}\left(Q_{\nu}-\lambda_{k} N_{k} Q_{\nu}\right)} \lambda_{k}^{d-1} E_{c e l l}^{M}\left(u_{0, \nu}, j+C_{M}\right)=0 .
$$

Appendix A. In this section we consider sets of finite perimeter in a bounded Lipschitz set $\Omega$ with assigned measure and prove a density result with sets having polyhedral boundary in $\Omega$ and maintaining the assigned measure.

Copyright $@$ by SIAM. Unauthorized reproduction of this article is prohibited. 
TheOrem A.1. Let $\Omega \subseteq \mathbb{R}^{d}$ be a bounded open set with Lipschitz boundary and let $m \in(0,1)$ be fixed. Then for any set $E$ with $|E|=m|\Omega|$ there exists a sequence $E_{n}$ such that $\partial E_{n} \cap \Omega$ is a polyhedral set, $\left|E_{n}\right|=m|\Omega|, \lim _{n \rightarrow+\infty}\left|E_{n} \triangle E\right|=0$, and $\lim _{n \rightarrow+\infty} \operatorname{Per}\left(E_{n}\right)=\operatorname{Per}(E)$.

Proof. Let us fix a set $E$ of finite perimeter and measure equal to $m|\Omega|$. By a classical density result (see $[11,17]$ ) we may select a sequence $\left(F_{n}\right)_{n}$ of sets with polyhedral boundary converging in measure and in perimeter to $E$. Then the measures of $F_{n}$ satisfy $\left|F_{n}\right|=m_{n}|\Omega|$ with $\lim _{n \rightarrow+\infty} m_{n}=m$ but in general $m_{n} \neq m$. In the sequel we will slightly modify the sets $F_{n}$ taking care that all the convergence and structure properties remain valid.

Let $0<\eta<1$ be fixed to be chosen later. By the regularity of $\Omega$ it is possible to find a positive $r>0$ and a finite set of disjoint cubes of side $r$ such that their union covers $\Omega$ up to an error less than $\eta$, that is, there exists a (finite) set $\mathcal{I}_{r}$ such that

$$
\left|\Omega \backslash \sqcup_{x \in \mathcal{I}_{r}} Q_{r}(x)\right| \leq \eta|\Omega| .
$$

We now claim that there exist $0<h<k<1$ and points $x_{h}, x_{k} \in \mathcal{I}_{r}$ such that

$$
\left|E \cap Q_{r}\left(x_{h}\right)\right|>h r^{d}, \quad\left|E \cap Q_{r}\left(x_{k}\right)\right|<k r^{d} .
$$

In fact, assuming by contradiction on $k, h$ that $\left|E \cap Q_{r}(x)\right| \leq h r^{d},\left|E \cap Q_{r}(x)\right| \geq k r^{d}$ for any $x \in \mathcal{I}_{r}$ we get

$$
m|\Omega|=|E| \geq \sum_{x \in \mathcal{I}_{r}}\left|E \cap Q_{r}(x)\right| \geq k \sum_{x \in \mathcal{I}_{r}} r^{d} \#\left(\mathcal{I}_{r}\right) \geq k(1-\eta)|\Omega|
$$

and

$$
\begin{aligned}
m|\Omega| & =|E|=\left|E \backslash \sqcup_{x \in \mathcal{I}_{r}} Q_{r}(x)\right|+\left|\sqcup_{x \in \mathcal{I}_{r}} E \cap Q_{r}(x)\right| \\
& \leq\left|\Omega \cap \sqcup_{x \in \mathcal{I}_{r}} Q_{r}(x)\right|+\sum_{x \in \mathcal{I}_{r}}\left|E \cap Q_{r}(x)\right| \leq|\Omega|-(1-h) r^{d} \#\left(\mathcal{I}_{r}\right) \\
& \leq h|\Omega|+\eta(1-h)|\Omega|=(h(1-\eta)+\eta)|\Omega| .
\end{aligned}
$$

Hence, according to fixing $\eta<\min \{m, 1-m\}$, we may select $h, k$ such that $0<$ $h(1-\eta)<m-\eta<m<k(1-\eta)$ and also $k<1$, and get a contradiction in both of the inequalities above.

Thus let $x_{k}, x_{h}$ be such that the inequalities in (A.1) hold for the initial set $E$. By taking into account the $L^{1}$ convergence of $F_{n}$ to $E$, a weak version of (A.1) is satisfied by $F_{n}$ for $n$ large enough, with $h, k$ replaced by $h / 2,(k+1) / 2$, i.e.,

$$
\left|F_{n} \cap Q_{r}\left(x_{h}\right)\right|>\frac{h}{2} r^{d}, \quad\left|F_{n} \cap Q_{r}\left(x_{k}\right)\right|<\frac{k+1}{2} r^{d} .
$$

In addition we also assume that $\left|m_{n}-m\right|<\min \left\{(h / 4) r^{d},(1-k) / 4 r^{d}\right\}$. We will modify $F_{n}$ in $Q_{r}\left(x_{k}\right)$ or alternatively in $Q_{r}\left(y_{k}\right)$, accordingly to the sign of the gap $m_{n}-m$.

Let us treat first the case in which $m_{n}<m$. Set $r_{n}=\left(4\left|m_{n}-m\right| /(1-k)\right)^{1 / d}$ and consider a collection of disjoint cubes $Q_{r_{n}}(y)$ of radius $r_{n}$ in $Q_{r}\left(x_{k}\right)$, intersecting along faces. We have that

$$
\sum_{Q_{r_{n}}(y) \subseteq Q_{r}\left(x_{k}\right)}\left|Q_{r_{n}}(y) \cap F_{n}\right| \leq\left|Q_{r}\left(x_{k}\right) \cap F_{n}\right|<\frac{k+1}{2} r^{d},
$$

Copyright (c) by SIAM. Unauthorized reproduction of this article is prohibited. 
hence, by the mean value theorem, taking into account that the cubes well-contained in $Q_{r}\left(x_{k}\right)$ are $\left[r / r_{n}\right]^{d}$, we can find $Q_{r_{n}}\left(y_{k}^{n}\right)$ such that, refining $h, k$ as above,

$$
\left|F_{n} \cap Q_{r_{n}}\left(y_{k}^{n}\right)\right|<\frac{k+3}{4} r_{n}{ }^{d} .
$$

Note that the function $\left(0, r_{n}\right) \ni t \rightarrow\left|Q_{t}\left(y_{k}^{n}\right) \backslash F_{n}\right|$ is continuous and, thanks to (A.2), surjective on the set $\left[0, m-m_{n}\right]$, so we may select $t_{n}$ such that $E_{n}=F_{n} \cup Q_{t_{n}}\left(y_{k}^{n}\right)$ satisfies the measure constraint. In the case $m_{n}>m$ we will argue analogously subdividing $Q_{r}\left(x_{h}\right)$ into small cubes with a slightly different radius $r_{n}^{\prime}$ depending on $h$ but still proportional to $\left|m_{n}-m\right|^{1 / d}$ and select $z_{h}^{n}, t_{n}^{\prime}$ such that $E_{n}=F_{n} \backslash Q_{t_{n}^{\prime}}\left(z_{h}^{n}\right)$ has mean volume fraction equal to $m$. Clearly, in both cases the regularity result of the boundary is maintained as well as the convergence in measure and in perimeter thanks to the fact that the additional perimeter of $E_{n}$ with respect to $F_{n}$ is contained in the boundary of a cube of radius $t_{n}$ or $t_{n}^{\prime}$ of order $\left|m-m_{n}\right|^{1 / d}$.

Remark A.2. By the locality of the construction above the same result holds for more general settings, for instance when $\Omega$ is substituted for by the $d$-dimensional flat torus. Actually in this last case it is enough to work locally on a single chart of the torus. Moreover, Theorem A.1 can be extended to a density result with prescribed measure also for functions assuming a finite set of fixed values, as for instance spin functions. Analogously one can replace the convergence of perimeters with suitable surface energies satisfying the hypotheses of Theorem 2.2. Note that in the case $\Omega$ is the whole space $\mathbb{R}^{d}$ or a cone, for example, the same result can be directly achieved by means of homothety arguments.

\section{REFERENCES}

[1] E. Acerbi, N. Fusco, And M. Morini, Minimality via second variation for a nonlocal isoperimetric problem, Comm. Math. Phys., 322 (2013), pp. 515-557.

[2] G. Alberti, G. Bouchitté, And P. Seppecher, Un résultat de perturbations singuliéres avec la norme $H^{1 / 2}$, C. R. Acad. Sci. Paris Sér. I Math., 319 (1994), pp. 333-338.

[3] R. Alicandro, A. Braides, And M. Cicalese, Phase and anti-phases boundaries in binary discrete systems: A variational viewpoint, Netw. Heterog. Media, 1 (2006), pp. 85-107.

[4] R. Alicandro and M. Cicalese, A general integral representation result for the continuum limits of discrete energies with superlinear growth, SIAM J. Math. Anal., 36 (2004), pp. $1-37$.

[5] R. Alicandro, M. Cicalese, and A. Gloria, Variational description of bulk energies for bounded and unbounded spin systems, Nonlinearity, 21 (2008), pp. 1881-1910.

[6] R. Alicandro, M. Cicalese, and A. Gloria, Integral representation results for energies defined on stochastic lattices and application to nonlinear elasticity, Arch. Ration. Mech. Anal., 200 (2011), pp. 881-943.

[7] R. Alicandro, M. Cicalese, and M. Ruf, Domain formation in magnetic polymer composites: An approach via stochastic homogenization, Arch. Ration. Mech. Anal., 218 (2015), pp. 945-984.

[8] L. Ambrosio And A. Braides, Functionals defined on partitions of sets of finite perimeter, I, J. Math. Pures Appl. (9), 69 (1990), pp. 285-305.

[9] L. Ambrosio and A. Braides, Functionals defined on partitions of sets of finite perimeter, II, J. Math. Pures Appl. (9), 69 (1990), pp. 307-333.

[10] L. Ambrosio, G. De Philippis, and L. Martinazzi, Г-convergence of nonlocal perimeter functionals, Manuscripta Math., 134 (2011), pp. 377-403.

[11] L. Ambrosio, N. Fusco, and D. Pallara, Functions of Bounded Variation and Free Discontinuity Problems, Oxford University Press, New York, 2000.

[12] G. Bouchitté, I. Fonseca, G. Leoni, and L. Mascarenhas, A global method for relaxation in $W^{1, p}$ and in $S B V_{p}$, Arch. Ration. Mech. Anal., 165 (2002), pp. 187-242.

[13] A. Braides, Г-Convergence for Beginners, Oxford Lecture Ser. Math. Appl. 22, Oxford University Press, Oxford, 2002. 
[14] A. Braides and A. Defranceschi, Homogenization of Multiple Integrals, Oxford University Press, New York, 1998.

[15] A. Braides and A. Piatnitski, Homogenization of surface and length energies for spin systems, J. Funct. Anal., 264 (2013), pp. 1296-1328.

[16] L. Caffarelli, J.-M. Roquejoffre, and O. Savin, Nonlocal minimal surfaces, Comm. Pure Appl. Math., 63 (2010), pp. 1111-1144.

[17] E. De Giongi, Nuovi teoremi relativi alle misure $(r-1)$-dimensionali in uno spazio a $r$ dimensioni, Ric. Mat., 4 (1955), pp. 95-113.

[18] G. Dal Maso, An Introduction to $\Gamma$-Convergence, Birkhäuser, Boston, 1993.

[19] L. Modica And S. Mortola, Un esempio di $\Gamma$-convergenza, Boll. Unione. Mat. Ital. B(5), 14 (1977), pp. 285-299.

[20] R. V. Kohn and P. Sternberg, Local minimizers and singular perturbations, Proc. Roy. Soc. Edinburgh Sect. A, 111 (1989), pp. 69-84.

[21] A. Giuliani, J. L. Lebowitz, And E. H. Lieb, Ising models with long-range dipolar and short range ferromagnetic interactions, Phys. Rev. B (3), 74 (2006), 064420.

[22] A. Giuliani, E. H. Lieb, And R. Seiringer, Realization of stripes and slabs in two and three dimensions, Phys. Rev. B (3), 88 (2013), 064401.

[23] T. Оhta AND K. KaWASAKI, Equilibrium morphology of block copolymer melts, Macromolecules, 19 (1986), pp. 2621-2632.

[24] O. Savin and E. VAldinoci, Г-convergence for nonlocal phase transitions, Ann. Inst. H. Poincarè. Anal. Non Linèaire, 29 (2012), pp. 479-500.

Copyright $@$ by SIAM. Unauthorized reproduction of this article is prohibited. 